\title{
Perspektiven des demokratischen Regierens und die Rolle der Politikwissenschaft im 21. Jahrhundert ${ }^{1}$
}

\author{
Michael Zürn
}

Perspectives of Democratic Governance and the Role of Political Science in the 21st Century Abstract: The core thesis of this contribution is that the primarily democratic sources of political legitimacy appear to be declining in significance. The crisis of parliamentary democracy in the West, the political and economic ascent of authoritatively constituted powers worldwide, the emergence of political authority beyond the nation state, and, connected to that, a corresponding rise in conflicting legal systems all point to the fact that underlying social and political developments lead to a shift within the set of established justifications of legitimate authority. In the course of present changes in the political landscape vis-à-vis sources of legitimacy like law, expertise, accountability, and collective identity, primarily democratic sources of political authority like participation and public discourse are beginning to fall by the wayside. Remarkably enough, these changes enjoy a high degree of public acceptance. These developments cause the democratic principle to become relativized in the course of deliberations over good political order; and, to that extent, the question of justification of legitimate authority and with it the question of democracy have been forced back onto the center stage of political controversies all over the world. This has far-reaching implications for political science as a formal discipline which indeed first began in Germany as Demokratiewissenschaft, the "science of democracy".

Keywords: Legitimacy, Democracies, Authoritarianism, International Institutions, Political Science

Schlagwörter: Legitimität, Demokratien, Autoritarismus, internationale Institutionen, Politikwissenschaft

1 Der Essay beruht auf einem Vortrag, den ich anlässlich der Festveranstaltung zum 60-jährigen Bestehen der Deutschen Vereinigung für Politische Wissenschaft am 14.5.2011 in Berlin gehalten habe. Ich danke den Teilnehmerinnen und Teilnehmern dieser Veranstaltung ebenso wie denen des TKI-Kolloquiums am Wissenschaftszentrum Berlin (WZB), insbesondere aber Rainer Forst, Mathias Koenig-Archibugi, Christoph Möllers, Rainer Schmalz-Bruns, Georg Simmerl und Thorsten Thiel für wertvolle Hinweise. 


\section{Einleitung}

Jugendaufruhr in London, brennende Autos in Berlin und Wutbürger in Stuttgart; eine globale Finanzkrise, der durch nächtliche Regierungstreffen begegnet werden soll, in denen ohne echte Beteiligung der Parlamente und mit einer gewissen Leichtigkeit Bürgschaften und Rettungsschirme in dreistelliger Milliardenhöhe beschlossen werden; der Aufstieg Chinas, der durch die Blockade im politischen System der USA beschleunigt und von Vielen mit einer nur wenig verhohlenen Bewunderung für dessen ökonomische Erfolge begleitet wird. Diese Ereignisse und Entwicklungen haben die Schlagzeilen im Sommer 2011 bestimmt. Nur auf den ersten Blick haben sie wenig gemeinsam. Sie verweisen aber tatsächlich allesamt darauf, dass die originär demokratischen Quellen der Legitimation von Herrschaft ihre hegemoniale Rolle zu verlieren drohen. Während die brennenden Autos und die Wutbürger eine wachsende Missachtung konventioneller Partizipationsformen zum Ausdruck bringen, verdeutlicht der Umgang mit der Eurokrise die Entmachtung der Parlamente, und der Aufstieg Chinas zeigt die Attraktivität eines undemokratischen Entwicklungsmodells in zumindest manchen Teilen der Welt.

Das Kernargument des Beitrags - dass die originär demokratischen Legitimationsquellen an Bedeutung zu verlieren scheinen - möchte ich wie folgt entfalten. In Abschnitt 3 werden die wichtigsten Quellen der Legitimation politischer Herrschaft in der Moderne identifiziert. Mithilfe dieser Legitimationskonzeption kann gezeigt werden, dass grundlegende gesellschaftliche und politische Entwicklungen zu einer Verschiebung innerhalb des Sets von etablierten Legitimationsfiguren führen. Originär demokratische Quellen politischer Herrschaft wie Partizipation und öffentliche Deliberation geraten im Zuge der gegenwärtigen Veränderungen der politischen Institutionenlandschaft gegenüber solchen Legitimationsquellen wie Recht und Expertise ins Hintertreffen. Bemerkenswerterweise finden diese Verschiebungen zumeist ein hohes Maß an gesellschaftlicher Akzeptanz. Die skizzierten Entwicklungen führen somit zu einer Relativierung des demokratischen Prinzips in der Auseinandersetzung über die gute politische Ordnung (Abschnitt 4).

Insofern rücken die Frage nach der Begründung von Herrschaft und damit auch die Demokratiefrage wieder in den Mittelpunkt politischer Auseinandersetzungen in der Welt (Abschnitt 5). Das hat weitreichende Implikationen für die Politikwissenschaft als Disziplin - in Deutschland ja als Demokratiewissenschaft ins Leben gerufen -, die ich in Abschnitt 6 erörtern möchte. Wer über das demokratische Regieren und die Rolle der Politikwissenschaft im 21. Jahrhundert spricht, sollte freilich vorab und gleichsam selbstreflexiv die Grenzen und Möglichkeiten von Aussagen über die Zukunft in der Politikwissenschaft ausloten. Damit möchte ich in Abschnitt 2 beginnen.

\section{Prognosen in der Politikwissenschaft}

Humphrey Littleton, ein britischer Jazzmusiker von Rang, antwortete einst auf die Frage nach der zukünftigen Entwicklung des Jazz: "Man, if I knew where Jazz was going, I would be there already!” Ganz ähnlich geht es dem gemeinen Politikwissenschaftler. 
Philip Tetlock hat dies in seiner bahnbrechenden Studie über „Expert Political Judgement" nachgewiesen (2005). Er brachte Ende der 1980er-Jahre 284 politische Experteninnen und Experten aller Art dazu, Vorhersagen über allgemeine politische Entwicklungen bis 2003 zu machen und hat die Daten nach allen Regeln moderner Methodik ausgewertet. Das Ergebnis ist für die Sozialwissenschaften wenig schmeichelhaft. Er schreibt:

"When we pit [the average of all, M. Z.] experts against minimalist performance benchmarks - dilettants, dart-throwing chimps, and assorted extrapolation algorithms - we find few signs that expertise translates into greater ability to make [good, M. Z.] forecasts” (Tetlock 2005, S. 20). ${ }^{2}$

Mit anderen Worten: Die durchschnittliche Prognosefähigkeit der Expertenschaft ist nicht besser als die von mit Wurfpfeilen versehenen Schimpansen.

Dennoch unterwirft sich Tetlock nicht dem wissenschaftstheoretischen Skeptizismus. Er sucht weiter und findet systematisch Varianzen, d. h. Merkmale von Expertinnen und Experten, die signifikant besser vorhersagen als das Zufallsprinzip. Erstaunlich ist dabei zunächst, was alles keinen Unterschied macht. So ist die mangelnde Prognosefähigkeit keinesfalls ein spezifisches Problem der Politikwissenschaft: Die Studie macht deutlich, dass es in dieser Frage keine systematischen Unterschiede zwischen Historikern, Ökonomen, Journalisten und Politikwissenschaftlern gibt. Andere Disziplinen sind also nicht besser. Weiterhin: Linke oder rechte politische Ausrichtung, Institutionalisten oder Realisten, Frauen oder Männer, mehr oder weniger Erfahrung - und übrigens auch die Frage des Doktortitels - sind allesamt irrelevant für die Prognosefähigkeit.

Was aber nun macht einen wirklichen Unterschied aus? Wer prognostiziert besser als der Durchschnitt? Zur Beantwortung dieser Frage identifiziert Tetlock unterschiedliche „kognitive Stile“ als relevant. Dabei benutzt er die Unterscheidung von Isaiah Berlin zwischen hedgehogs und foxes (vgl. Berlin 1953). „Igel“ wissen meistens „eine große Sache“, weiten die Erklärungsreichweite ihrer Theorie zumeist in deduktiver Weise aus, arbeiten nicht selten mit anspruchsvollen methodischen Instrumenten und sind voller Selbstvertrauen, was die Prognosefähigkeit ihrer Theorie anbetrifft. Sie machen aber schlechte Vorhersagen. „Füchse“ hingegen wissen viel Unterschiedliches, changieren permanent zwischen Theorie und Empirie, bekennen sich zu einem gewissen Grad zum analytischen Eklektizismus und sind skeptisch, was ihre eigene Prognosefähigkeit anbetrifft. Füchse prognostizieren aber deutlich besser als der Durchschnitt der Experten. Um nochmals Tetlock zu zitieren:

"The hedgehog-fox dimension did what none of the 'content' measures of political orientation, and none of the measures of professional background could do: distinguish observers of the contemporary scene with superior forecasting records, across regions, topics and time" (Tetlock 2005, S. 75).

2 Im Originalzitat wird von "either 'well-calibrated' or 'discriminating' forecasts” gesprochen. Für den vorliegenden Zweck ist diese Ausdifferenzierung von zwei Dimensionen guter Vorhersagen nicht zentral. 
Freilich bleiben Prognosen ein schwieriges Geschäft. Wenn man sich dennoch auf das Feld der Zukunftsaussagen begibt, dann sollte man jedenfalls nicht einfach eine Theorie mit Tunnelblick zur Anwendung bringen, sondern verschiedene Entwicklungen und Theorien ins Auge fassen, sie so gut wie möglich miteinander verbinden und dabei Vereinseitigungen und radikale Vereinfachungen vermeiden. Ich möchte dies im Folgenden tun, indem ich zunächst einen konzeptionellen Rahmen zum Verständnis politischer Legitimität in der Moderne anbiete, um vor diesem Hintergrund vier demokratierelevante Entwicklungen im Zeitalter der Globalisierung zu analysieren.

\section{Legitimation politischer Herrschaft heute}

Legitimität verweist darauf, dass etwas legitim ist, während Legitimation Aktivitäten oder Prozesse bezeichnet, die etwas legitim machen. Legitimität wird über Legitimationsprozesse, also durch Kommunikation über Legitimität erzeugt (vgl. auch Nullmeier et al. 2010, Kap. 1). Dabei können sich Legitimität und Legitimation auf unterschiedliche Objekte beziehen. Die Begriffe können beispielsweise genutzt werden, um Verhaltensweisen zu bezeichnen, die mit den normativen Erwartungen einer Gesellschaft kompatibel sind („eine legitime Reaktion“) oder eine Bewertung von kommunikativen Akten vornehmen („ein legitimes Argument"). Je nach Objektbereich gelten unterschiedliche Legitimationsstandards und -quellen. Mir geht es im Folgenden um politische Legitimität im engeren Sinne als ein Merkmal herrschaftsausübender politischer Institutionen und Ordnungen - also um den Weberschen Legitimitätsbegriff.

Politische Legitimität und Legitimation können dabei sowohl auf die normative Anerkennungswürdigkeit einer politischen Ordnung und ihrer Rechtfertigung verweisen (siehe Habermas 1973, Teil III), als sich auch rein deskriptiv auf deren soziale Akzeptanz (den Legitimitationsglauben, vgl. Weber 1968) beziehen. Zusammengenommen heißt das, wenn Herrschaft auf der Grundlage von anerkennungswürdigen Normen ausgeübt wird und auf ein gewisses Maß an Zustimmung stößt, kann sie als legitim gelten.

Diese inhärent doppelte Kodierung des Konzepts (normativ und empirisch) muss auch in der empirischen Forschung ihren Niederschlag finden. Politische Herrschaft kann in diesem Sinne dann als legitim angesehen werden, wenn die durch sie produzierten kollektiv bindenden Normen und Regeln auf geteilten Überzeugungen über das Gemeinwohl (bzw. das öffentliche Interesse) sowie auf geteilten Vorstellungen prozeduraler Fairness beruhen. Es geht also nicht um die unmittelbare Akzeptanz, die eine Ordnung genießt, sondern um die Frage, ob und inwieweit die Herrschaftspraktiken mit normativen Grundprinzipien kompatibel sind, die in der betreffenden Gesellschaft geteilt und verankert sind: "a given power relationship is not legitimate because people believe in its legitimacy, but because it can be justified in terms of their beliefs" (Beetham 1991, S. 11).

Eine solche Konzeption politischer Legitimität unterscheidet sich von einer rein normativen Theorie guter Ordnung oder gerechter Gesellschaft, aber auch von einer rein empirischen Erforschung der sozialen Akzeptanz politischer Systeme. Neben der philosophischen Rechtfertigung und der sozialen Akzeptanzforschung 
bilden legitimationswirksame Strukturbildungen eine eigenständige Sphäre, deren Betrachtung notwendig ist, um ein angemessenes Gesamtbild zu erlangen und um die Brücke zwischen Normativität und Empirie schlagen zu können. ${ }^{3}$ So lange wie die Grundüberzeugungen einer Gesellschaft über das öffentliche Interesse und prozedurale Fairness nicht drastisch verletzt werden, kann in diesem Sinne eine politische Ordnung auch dann als legitim angesehen werden, wenn ihre konkrete Funktionsweise normative Defizite aufweist. Umgekehrt war die Gleichsetzung von sozialer Akzeptanz und Legitimität auch nie im Sinne von David Easton, in dessen Modell die Legitimität einer politischen Ordnung 4 nur ein Einflussfaktor für die Erklärung von Akzeptanz ist (1975, S. 445-447). ${ }^{5}$ Generell erscheint mir die Rezeption von Weber, die Legitimität auf die soziale Akzeptanz einer politischen Ordnung reduziert, nur bedingt nachvollziehbar. Auch für ihn ist eine legitime Ordnung gerade dadurch gekennzeichnet, dass sie eine über das Zweckrationale hinausgehende Motivation habe und insofern Geltung besäße. Es geht also auch bei ihm um den Glauben über das im politischen Raum normativ Richtige. Insofern kann der Legitimitätsanspruch politischer Ordnungen durchaus durch empirische Legitimitätsurteile hindurch greifen und dadurch Raum für Ideologiekritik und eine rekonstruktive Theorielogik schaffen. Eine einseitig auf den Legitimitätsglauben abhebende Konzeption beraubt hingegen die Sozialwissenschaften der Möglichkeit, kritische Urteile zu treffen, wenn die Akzeptanzindikatoren hoch sind. So wäre es nämlich dann konzeptionell ausgeschlossen, die frühen Jahre nationalsozialistischer Herrschaft als illegitim zu beschreiben.

Zudem entledigt sich die Legitimationsforschung mit einer einseitig auf den Legitimitätsglauben abhebenden Konzeptualisierung der relevanten Fragen zum Verhältnis von normativen Prinzipien und sozialer Akzeptanz: Welche normativen Prinzipien dienen in welchem Maße und in welchem Kontext als Quelle der Legitimation? Das hier skizzierte Verständnis politischer Legitimität erlaubt es hingegen nach der empirischen Relevanz unterschiedlicher Quellen der Legitimation zu fragen. Folglich muss Legitimität als eine Variable begriffen werden, die nicht binär zu fassen ist.

Mit Blick auf die empirische Analyse der Quellen politischer Legitimität gehe ich zunächst davon aus, dass der Appell an das öffentliche Interesse bzw. das Gemeinwohl des regulierten Kollektivs die Grundlage legitimer politischer Ordnungen darstellt. Selbst traditionelle Begründungen von Herrschaft beruhten auf Begründungsmustern, die das Gemeinwohl betonen. Der Legitimitätsglaube

3 In diesem Sinne greift m. E. auch die konstruktivistisch motivierte Legitimationsforschung, wie sie in einflussreichen Teilen der Internationalen Beziehungen vertreten wird, zu kurz. Dort verweist „Legitimität" auf die internalisierte Einstellung, dass einer Institution oder einer Norm gefolgt werden soll (vgl. Hurd 2007, S. 7 und Kap. 2).

4 Easton (1975, S. 451) definiert Legitimität ganz in unserem Sinne „[...] as the conviction that it is right and proper $[\ldots]$ to accept and obey the authorities and to abide by the requirements of the regime. It reflects the fact that in some vague or explicit way a person sees these objects as conforming to his own moral principles, his own sense of what is right and proper in the political sphere."

5 Anders übrigens Seymour Martin Lipset (1960, S. 64): "Legitimacy involves the capacity of a political system to engender and maintain the belief that existing political institutions are the most appropriate and proper ones for the society." 
verflüchtigt sich sofort, wenn die Herrschenden als exklusiv eigennützig und selbstsüchtig wahrgenommen werden. Das ist genau die feine Linie, die den Unterschied ausmacht, etwa zwischen den frühen Jahren des ägyptischen Autoritarismus von Nasser, als die Gemeinwohlbindung halbwegs plausibel erschien, und den späten Mubarak-Jahren, in denen entsprechende Appelle als leere Floskeln verpufften.

Mit der Entscheidung das Gemeinwohl bzw. das öffentliche Interesse als Anker von Legitimationsprozessen zu setzen, verbindet sich die Überzeugung, dass es für eine empirische Analyse mehr als nur demokratische Legitimation politischer Herrschaft geben kann. Die Ableitung der Legitimität exklusiv aus dem Prinzip der individuellen und kollektiven Selbstbestimmung mag für eine normative Analyse angemessen sein, für eine empirische Analyse schränkt sie aber Legitimationsforschung auf einen bestimmten historischen Zeitraum in einer bestimmten Region der Welt ein. Gleichzeitig schließt dieses Verständnis nicht aus, dass im Sinne der oben skizzierten rekonstruktiven Logik der Legitimitätsanspruch politischer Ordnungen durch empirische Legitimitätsurteile hindurch greifen kann und sich dadurch eine kritische Perspektive in demokratischer Absicht eröffnet (vgl. hierzu Abschnitt 5).

Der bloße Verweis auf das Gemeinwohl reicht als Legitimationsstrategie freilich nicht aus. Die entsprechende Behauptung muss durch Verweise auf Quellen und Verfahren, mittels derer Legitimität beansprucht werden kann, gedeckt sein. Auf einer ersten, sehr allgemeinen Ebene geht es zunächst um grundlegende Prinzipien. Zum einen beinhaltet jede Ausübung politischer Herrschaft eine stratifikatorische Differenzierung: es gibt Entscheidungsträger und Entscheidungsnehmer. Diese stratifikatorische Differenzierung muss in einer modernen politischen Ordnung rational gerechtfertig werden - Tradition und Vererbung können nicht mehr als Legitimation dienen, Leistung und Kompetenz zählen stattdessen. Zum Zweiten muss eine legitime politische Ordnung transparente Regeln besitzen. Die beliebige und intransparente Ausübung von Herrschaft untergräbt jeden Legitimationsanspruch. Insofern ist "rule by (transparent) law" eine weitere basale Grundbedingung legitimer Herrschaft, die gleichsam unhintergehbar ist. Ein rationales Prinzip der stratifikatorischen Differenzierung plus Legalität reichen natürlich unter normalen Umständen nicht zur Legitimation einer politischen Ordnung aus. Diese allgemeinen Prinzipien bilden bestenfalls notwendige Bedingungen legitimer Herrschaft.

Das führt zu einer zweiten Ebene von spezifischeren Legitimationsquellen wie Expertise, Wohlstandsmehrung oder demokratische Partizipation. Die Legitimationsquellen können sich dabei sowohl auf den Prozess der Entscheidungsfindung als auch auf den Gehalt einer Entscheidung oder Norm beziehen. ${ }^{6}$ Sie sind innerhalb der Moderne kontextspezifisch im Sinne der „multiple modernities“ (Shmuel Eisenstadt). Sie können also in ihrer Kombination und Zusammensetzung je nach Ausprägung der gesellschaftlichen Überzeugungen und je nach Art der ausgeübten Herrschaft variieren. Die verschiedenen Legitimationsquellen sind in einem

6 Dies verweist natürlich auf die vielgenutzte Unterscheidung zwischen Input- und Outputlegitimität (Scharpf et al. 1976; Scharpf 1999, Kap. 1). 
gewissen Rahmen auch gegenseitig substituierbar, d. h., sie müssen nicht alle gleichzeitig vorhanden sein, nicht jede einzelne Legitimationsquelle ist immer notwendig. Die Bedingungen angemessener Legitimation sind in dieser reflexiven Konzeption selbst Teil der Auseinandersetzung über die Legitimität politischer Herrschaft. ${ }^{7}$

Welche Herrschaftspraktiken sind dazu geeignet das öffentliche Interesse bzw. das Gemeinwohl des regulierten Kollektivs zu befördern und können insofern als legitimitätsstiftend gelten? Was sind also die spezifischen Quellen politischer Legitimation in der Moderne? Ihr gemeinsamer Nenner ist, dass sie plausiblerweise den Anspruch erheben können, das Gemeinwohl bzw. das öffentliche Interesse zu befördern. Daher haben v. a. solche Entscheidungsverfahren und Politikergebnisse eine Chance als legitim angesehen zu werden, die als unparteiisch und neutral gelten können. Unvoreingenommene, nicht von vornherein eine Seite oder eine gesellschaftliche Gruppe bevorzugende Verfahren und Praktiken können potenziell Legitimität herstellen. Besonders deutlich wird dies im Extremfall der Lotterie. Obgleich das der Lotterie inhärente Zufallsprinzip der politischen Anforderung der abwägenden Entscheidung in gewisser Weise entgegensteht, kann die Lotterie gerade aufgrund ihrer maximalen Unvoreingenommenheit das Prinzip der politischen Gleichheit und Fairness befördern, wenn knappe Güter oder Positionen verteilt werden (siehe Buchstein 2009).

Vor diesem Hintergrund erscheinen mir empirisch betrachtet v. a. sechs spezifische Rechtfertigungen für politische Herrschaft grundlegend zu sein. Diese Liste der sechs potenziellen Quellen politischer Legitimität leitet sich aus dem Gemeinwohlprinzip und der damit verbundenen Unparteilichkeitsanforderung ab. ${ }^{8}$

- Gute Politikergebnisse beruhend auf unvoreingenommener Expertise und Sachkenntnis: Die Expertise leitet sich von einer Idee der Wissenschaft als unabhängige, keine Rücksicht auf Partikularinteressen nehmende Wahrheitssuche ab, die auf systematischen Methoden beruht. Damit verbindet sich die Hoffnung auf erfolgreiche, zielführende Politiken, die insbesondere die Wohlfahrt einer Gemeinschaft befördern, also das, was Fritz Scharpf (1991, S. 621628) primär unter Output-Legitimität versteht und auf die epistemische Qualität politischer Entscheidungen verweist.

- Die Beförderung des Selbstwertgefühls der Gemeinschaft der Regelungsadressaten ist eine zweite Form eines weit gefassten Verständnisses von OutputLegitimität. Die Herstellung einer vorgestellten Gemeinschaft (Anderson 1983) und die Förderung des kollektiven Selbstwertgefühls geschehen nicht

7 Vgl. zu diesem Aspekt der Konzeptualisierung von Legitimität insbes. Nullmeier et al. (2010). Ob sich daraus implizit eine gleichsam reflexive Hegemonie demokratischer Legitimation ergibt - so ein Hinweis von Rainer Schmalz-Bruns -, hängt von der Demokratiedefinition und dem Ausmaß der Vermachtung der Auseinandersetzung über politische Legitimität ab.

8 Diese Liste der zentralen Legitimationsquellen der Moderne hat einen enumerativen Charakter. Sie beansprucht zum einen nicht zwingend vollständig zu sein. So ist beispielsweise die Lotterie als Legimitationsquelle nicht genannt, da sie empirisch als Legitimation kaum genutzt wird. Zum anderen entspringt diese Liste auch keiner typologischen Logik im strikten Sinne. Vgl. aber Halberstam (2009), der aus der Konstutionalisierungslogik drei Quellen politischer Legitimität als abgeschlossene Liste entwickelt: „voice“, „rights“ und „expertise“. 
selten durch scharfe Grenzziehungen zwischen Innen und Außen, was es zur, normativ betrachtet, problematischsten Legitimationsquelle macht.

- Der Schutz der Grundrechte und die Beförderung der Rechtsgleichheit stellen eine dritte Quelle von Output-Legitimität dar. Der Rekurs auf die Herrschaft des Rechts (Tamanaha 2004) lässt sich aus dem Grundwert der Autonomie des Individuums ableiten.

- Die Rechenschaftspflichtigkeit der Herrschenden - accountability - stellt eine erste verfahrensorientierte Legitimationsquelle im Sinne der Input-Legitimität dar (vgl. z. B. Grant u. Keohane 2005). Diese darf aber nicht mit Demokratie verwechselt werden. Rechenschaftspflichtigkeit erfordert nicht die Partizipation der Regelungsbetroffenen bei spezifischen Entscheidungen, sie macht nur die Entscheidungsträger für die von ihnen getroffenen Entscheidungen rechtlich und/oder elektoral verantwortlich.

- Gleiche Partizipationschancen aller Regelungsbetroffenen oder zumindest aller Regelungsadressaten: Die Partizipation kann direkt oder durch Repräsentation erfolgen. Repräsentanz kann wiederum funktional oder territorial organisiert sein. In der institutionellen Ausgestaltung können also Partizipations- und Repräsentationsmechanismus vielfältig und stark unterschiedlich sein. Sie setzen aber jeweils an einem zentralen Aspekt des demokratischen Prinzips an: Alle, die durch eine Entscheidung betroffen sind oder adressiert werden, sollten bei dieser Entscheidung mitwirken können (Dahl 1989)..$^{9}$

- Schließlich verweist die deliberative Demokratietheorie auf eine weitere prozedurale Legitimationsressource: öffentlicher Diskurs und Kontestation. Sie beruht auf der normativen Überzeugung, dass die Aggregation der Interessen im Zuge der Partizipation oder Repräsentation durch eine offene Auseinandersetzung über das Gemeinwohl begleitet werden muss (Habermas 1994).

Mithilfe dieses Portfolios an Legitimationsquellen politischer Herrschaft ist nun zu zeigen, dass vier zentrale Entwicklungen unserer Zeit allesamt eine Wegentwicklung von den beiden originär demokratischen Prinzipien politischer Legitimation - nämlich Partizipation und öffentliche Auseinandersetzung - beinhalten. Insofern muss - so die Kernthese - von einer Relativierung des demokratischen Prinzips in der Auseinandersetzung über gute politische Ordnungen gesprochen werden. Wenn sich diese These bestätigt, dann verweist dies gleichzeitig darauf, dass die relative Bedeutung der genannten Legitimationsquellen selbst Resultat von Auseinandersetzungen über politische Legitimität ist.

\section{Vier Trends politischer Legitimation}

In diesem Abschnitt möchte ich vier aktuelle und demokratierelevante Entwicklungen ausmachen. Sie sind aktuell, da sie allesamt historisch betrachtet relativ neu sind und sich gegenwärtig manifestieren. Sie sind demokratierelevant, da sie

9 Die Formulierung „Regelungsbetroffenen oder Regelungsadressaten “ deutet an, dass die Konkretisierung dieses Prinzips selbst umstritten ist - nicht aber das Prinzip als solches. 
auf Probleme der legitimen Herrschaftsausübung verweisen und mithin die Frage nach der demokratischen Legitimation wieder verstärkt in den Fokus politischer Auseinandersetzungen rücken. Es geht um die Diskussion über die Krise der parlamentarischen Demokratie im Westen, um den weltpolitischen und ökonomischen Aufstieg von außereuropäischen Mächten, um die Entstehung politischer Autorität jenseits des Nationalstaates sowie um die damit verbundene Entstehung konfligierender Rechtsordnungen. Hinter diesen abstrakt gefassten Entwicklungen verbergen sich konkrete und gleichsam tagesaktuelle Großproblemlagen: die Krise westlicher Demokratien, wie sie sich etwa in Unruhen in englischen Städten zeigte, der Aufstieg eines autoritär geführten Chinas, die zunehmende Eingriffstiefe internationaler Institutionen in ehedem innere Angelegenheiten, wie etwa zuletzt in Griechenland oder Libyen, und zunehmende Kompetenzstreitigkeiten zwischen unterschiedlichen politischen Ordnungen, wie sie sich öffentlich zuletzt im Lissabon-Urteil des Bundesverfassungsgerichts äußerten.

Die Zusammenschau dieser Entwicklungen entspringt in doppelter Hinsicht einer globalisierten Perspektive. Sie unterteilt den globalen politischen Raum von Innen nach Außen blickend in vier Bereiche und bricht dabei die Logik des methodologischen Nationalismus bzw. der segmentären Differenzierung der politischen Räume auf: die nationalen politischen Systeme der westlichen Welt, nationale politische Systeme außerhalb der westlichen Welt, globale politische Institutionen und schließlich das Zusammenspiel unterschiedlicher politischer Ordnungen in einer globalen Mehrebenenperspektive. Zudem sind all die genannten Entwicklungen direkte oder indirekte Folgen gesellschaftlicher Denationalisierungsprozesse und der damit verbundenen Gefahren und Chancen. Die Befunde zur Krise der westlichen Demokratie werden nicht selten mit ökonomischen Entgrenzungsprozessen in Verbindung gebracht, der Aufstieg Brasiliens, Chinas, Indiens und anderer Mächte außerhalb des nordatlantischen Raums kann ebenso als Folge ökonomischer Globalisierungsprozesse gedeutet werden. Internationale Institutionen entstehen vor dem Hintergrund denationalisierter Problemlagen und verursachen Kompetenzkollisionen. Insofern lässt sich mit guten Gründen erwarten, dass die skizzierten Entwicklungen auch in nächster Zukunft - ich rufe freilich die oben aufgestellten ,Prognosewarnschilder' in Erinnerung - Bestand haben werden.

\subsection{Die Legitimationskrise majoritärer politischer Institutionen}

Die vergleichende Demokratieforschung hat in den letzten Jahrzehnten einen Rückgang politischer Beteiligung diagnostiziert, der sich im Durchschnitt der OECD-Länder in einer seit Ende der 60er-Jahre kontinuierlich sinkenden Wahlbeteiligung und einem deutlichen Rückgang der Parteimitgliedschaften zeigt (Hay 2007, S. 21).

Kritisch wird der Befund insbesondere dann, wenn diese Entwicklung durch ein geringes Vertrauen in Parteipolitiker, die Regierung und das Parlament begleitet wird. So zeigt beispielsweise die jüngste Erhebung von Infratest dimap (2009) zum Institutionenvertrauen in Deutschland genau dieses: „Sehr großes“ oder „großes“ Vertrauen in die politischen Parteien haben laut dieser Befragung nur 23 
Prozent der Deutschen, womit diese auf dem letzten Platz der abgefragten Institutionen und sogar noch hinter den Medien und Arbeitgeberverbänden rangieren. Der Bundestag erreicht mit 48 Prozent einen durchschnittlichen Wert. ${ }^{10}$ Hay (2007, S. 34) zeigt auf der Grundlage von Daten des Harris Poll und des Eurobarometer (jeweils 2004), dass diese Entwicklung nicht deutschlandspezifisch ist. Unter einer Vielzahl an öffentlichen Institutionen erhalten die Parteien in den USA und der EU durchweg die schlechtesten Vertrauenswerte, schlechter noch als große Unternehmen und die Medien. Auch die Parlamente befinden sich in der Negativhitliste weit hinten, im Allgemeinen auf dem viertletzten Rang von zwölf öffentlichen Institutionen.

Vor diesem Hintergrund scheinen die aktuellen Thesen von der Legitimationskrise westlicher Demokratien besser begründet als die von den Legitimationsproblemen des politischen Systems im Spätkapitalismus in den 70er-Jahren (vgl. v. a. Habermas 1973; Offe 1972). Insbesondere das Theorem von der Postdemokratie (Crouch 2008), aber auch die agonale Demokratietheorie (Mouffe 2007) beklagen die Entleerung politischer Prozesse in den Staaten der westlichen Welt. Demnach stehen auf der einen Seite immer raffiniertere Mechanismen zur Manipulation der öffentlichen Meinung und auf der anderen immer farblosere und oberflächlichere Parteiprogramme, die sich nur noch in der Tönung, kaum aber in der Substanz unterscheiden. Das Lebenselixier der demokratischen Institutionen, nämlich die grundlegenden Widersprüche zwischen konkurrierenden Interessenlagen, verflüchtige sich dadurch. Stattdessen würden Sachorientierung und diskursive Konsensfindung hochgehalten. Dahinter verberge sich aber nichts anderes als die Zunahme des Einflusses privilegierter Eliten, die sich von den demokratischen Prozessen abschotten. ${ }^{11}$

Die Betrachtung zusätzlicher Daten relativiert dieses Bild jedoch erheblich und zeigt, dass das Vertrauen in das politische System als Ganzes nach wie vor hoch ist. Nach den Daten der European Election Study ${ }^{12}$ ist die große Mehrheit der

10 Auch die aktuellen Daten des European Social Survey zeigen, dass eine frappierende Abwesenheit von Vertrauen in Parteien und auch Parlamente in allen konsolidierten Demokratien Europas besteht.

11 Das entsprechende Argument ist von Colin Hay (2007, S. 159) sehr luzide formuliert worden: "I have suggested that privatization, the contracting-out of public services, the marketization of public goods, the displacement of policy-making autonomy from the formal political realm to independent authorities, the rationalization and insulation from critique of neoliberalism as an economic paradigm, and the denial of policy choice (for instance, in discerning the imperatives of competitiveness in an era of globalization) are all forms of depoliticization. Each serves, effectively, to diminish and denude the realm of formal public deliberation; each might be seen as a legitimate cause of political disaffection and disengagement (at least from formal politics). Moreover, the increasing adoption of a range of electoral competition (at least in policy terms), the depoliticization of whole areas of public policy, a tendency to remove questions of policy content, especially those involving significant institutional reform from electoral scrutiny, and a compensating politicization of the personality traits of candidates for high office." Crouch, Hay u. a. erklären also die nachlassende Partizipation mit Merkmalen des politischen Prozesses. Dem gegenüber stehen Ansätze, die v. a. gesellschaftliche Änderungen für den Niedergang politischer Partizipation verantwortliche machen. Allen voran Robert Putnams (2000) These vom schwindenden Sozialkapital, die allerdings mit Blick auf Europa empirisch kaum tragfähig ist.

12 Antworten auf die Frage „How satisfied are you, on the whole, with the way democracy works in [your country; M. Z.]?“ in der European Election Study 2009 (PIREDEU 2010). 
Bürgerinnen und Bürger nach wie vor insbesondere im Nordwesten Europas mit dem Funktionieren der Demokratie zufrieden - mit Spitzenwerten von beispielsweise 92 Prozent in Dänemark, 88 Prozent in Luxemburg und 81 Prozent in Schweden. Wenn man zusätzlich das an der Universität Zürich und dem Wissenschaftszentrum Berlin (WZB) entwickelte Demokratiebarometer zu Rate zieht, so zeigt sich, dass insbesondere in den Ländern, denen vom Barometer eine hohe demokratische Qualität attestiert wird, - freilich mit einigen bemerkenswerten Abweichungen - auch die Zufriedenheitswerte hoch sind (vgl. Bühlmann et al. 2011). Zudem zeigen langfristige Datenreihen (insbes. Eurobarometer und World Values Survey), dass in westlichen Ländern das normative Prinzip der Demokratie nach wie vor volle Zustimmung findet.

Es zeigt sich also, dass weder die generelle Unterstützung für die nationalen politischen Systeme (i. S. von David Easton) noch die für das normative Prinzip der Demokratie in den westlichen Ländern über Zeit einen deutlichen Rückgang erfahren haben (vgl. auch Norris 2011, Kap. 4 und 5). Deshalb ist es überzogen von einer generellen Systemkrise oder einer „Post“demokratie zu sprechen.

Es lässt sich jedoch eine bemerkenswerte interne Verschiebung der zentralen Legitimationsquellen in den westlichen Demokratien beobachten. Es zeigt sich nämlich ein deutlicher Bedeutungsverlust etablierter demokratischer Verfahren sowohl auf der Ebene der Einstellungen als auch mit Blick auf die politischen Entscheidungsprozesse. Auf der Seite der Einstellungen korrespondiert die skizzierte Unzufriedenheit mit den Kerninstitutionen der parlamentarischen Demokratie wie den Parlamenten und den Parteien mit einer beachtlichen Wertschätzung von nicht-majoritären Institutionen. Während also die Kerninstitutionen der Mehrheitsbildung, die den Bereich der Politik im engen Sinne definierten, äußerst kritisch beäugt werden, haben die Institutionen mit gesamtgesellschaftlicher Entscheidungskompetenz, die dem politischen Prozess entzogen sind, wie etwa die Zentralbanken oder die Verfassungsgerichte, überall ein deutlich höheres Ansehen als die demokratischen Kerninstitutionen. So ist in allen 22 Ländern, die im European Social Survey 2008 erfasst sind, das Vertrauen in das Rechtssystem höher als in das Parlament und die Parteien (Norwegian Social Science Data Services 2011). Gleiches gilt für die entsprechenden Eurobarometer-Daten, wo das Rechtssystem allerdings nochmals deutlich von der Polizei und dem Militär geschlagen wird. ${ }^{13}$

Das allgemeine Bild wird durch die enorme Wertschätzung bestätigt, die den bekanntesten nicht-majoritären Institutionen im politischen System der Bundesrepublik entgegengebracht wird. Die Daten von Allensbach über das Ansehen politischer Akteure im Vergleich zeigen, dass seit 1985 kontinuierlich Bundespräsident, Bundesverfassungsgericht und Bundesbank an der Spitze stehen, Bundestag, Bundesrat und Bundesregierung am Ende (Allensbacher Jahrbuch der Demoskopie [1974-1999], zit. nach Kneip [2009, S. 201]). Aktuellere Daten aus dem Jahre

13 Ein ähnliches Bild zeigen die Vertrauenswerte in unterschiedliche Berufsgruppen. Während beispielsweise in Großbritannien Doktoren, Lehrer sowie Wissenschaftler im Gesamtfeld vorne liegen, führen Richter klar innerhalb der Berufe, die im politischen System im weiteren Sinne tätig sind - mit 60 Prozent mehr positiven Einschätzungen als negativen; gefolgt von den Polizisten (+ $26 \%$ ). Das Schlusslicht im gesamten Feld bilden gemeinsam Minister, Politiker und Journalisten mit 51 Prozent mehr negativen Einschätzungen als positiven (Hay 2007, S. 35). 
2006 deuten darauf hin, dass sich der Trend akzentuiert hat: Während dem Bundespräsidenten und dem Bundesverfassungsgericht über 70 Prozent der Befragten „eher vertrauen“, überwiegt gegenüber Bundestag, Bundesregierung und Parteien die Zahl derer, die diesen Institutionen „eher kein Vertrauen“ entgegenbringen, deutlich. ${ }^{14}$

Diese Einstellungsdaten finden eine erstaunliche Entsprechung in der institutionellen Realität. Die Bedeutung nicht-majoritärer Institutionen, die kollektive verbindliche Entscheidungen treffen und mithin politische Autorität ausüben, hat gleichfalls deutlich zugenommen. So kann über die letzten Jahrzehnte eine uniforme Bedeutungszunahme von unabhängigen Zentralbanken in der westlichen Welt beobachtet werden. Sie wurden nach und nach in vielen Ländern - und dann auch mit der Europäischen Zentralbank EU-weit - eingeführt und in ihrer Unabhängigkeit gestärkt. Zwischen 1990 und 2008 haben sage und schreibe 84 Länder Gesetze verabschiedet, mittels derer die formale Autonomie der Zentralbanken gestärkt wurde (Rapaport et al. 2009; siehe dazu auch Cukierman 2008). Gleichzeitig wuchs im Zuge des Monetarismus die Bedeutung des geldpolitischen Instrumentariums im Gesamtwerkzeugkasten der ökonomischen Steuerung an.

Die Zentralbanken sind aber letztlich nur Ausdruck einer breiteren Entwicklung: der Zunahme von sog. „independent agencies" (Shapiro 1997; Shapiro 2005; Gilardi 2008). Im Schnitt sind inzwischen laut einer quantitativen Studie in 73 Prozent Prozent aller Politikfelder der untersuchten Länder „autonomous regulatory agencies“ tätig (Jordana et al. 2011). Die Studie weist zwischen 1966 und 2007 ein stetiges quantitatives Wachstum nach, das besonders in den 1990erJahren beinahe exponentielle Züge erreichte und erst im neuen Jahrtausend wieder nachließ.

Mindestens genauso wichtig ist die Bedeutungszunahme von Verfassungsgerichten. Ran Hirschl (2004, S. 1) fasst diese Entwicklung konzise im ersten Satz seiner kritischen Analyse „Towards Juristocracy“ zusammen:
"Over the past few years the world has witnessed an astonishingly rapid transition to what may be called juristocracy. Around the globe, in more than eighty countries and in several transnational entities constitutional reform has transferred an unprec- edented amount of power from representative institutions to judiciaries.”

Für ihn ist diese Form der judicial empowerment eine Strategie herrschender politischer Eliten, um die eigene Hegemonie bewahren zu können (Hirschl 2004, Kap. 2). Für Alec Stone Sweet (2000) sind die allseits zu beobachtenden Prozesse der Stärkung konstitutioneller Normen und Gerichte gar Ausdruck eines inhärenten Selbstverstärkungsmechanismus innerhalb moderner politischer Systeme, der dem generellen Trend von der dyadischen zur triadischen Konfliktbewältigung entspricht.

Durch die Europäische Integration hat der Trend eine zusätzliche Verstärkung erfahren. Dafür führt Daniel Kelemen (2011, S. 23) zwei Gründe an:

14 Unveröffentlichte repräsentative Forsa-Umfrage unter 2017 Bundesbürgern für das Wissenschaftszentrum Berlin für Sozialforschung, Abteilung Demokratieforschung im November 2006, zitiert nach Kneip (2009, S. 202). 
"First, the EU's drive to create a single market has led to the dismantling of many traditional, national regulatory regimes and to their replacement with far more formal and judicialized regulatory regimes at the EU level. [...] Second, the high degree of political fragmentation that is so deeply embedded in the EU's institutional structure has generated powerful political incentives for policy makers to rely on adversarial legalism as mode of governance."

Dies habe zu einer europäischen Variante des aus den USA bekannten „adversarial legalism“ geführt, dem „Eurolegalism“. In der Folge werden Konflikte über die Entwicklung und die Anwendung von Normen zunehmend vor Gerichten ausgetragen. Man denke nur an Beispiele wie die Antidiskriminierungsurteile des EuGH, die Stärkung der Konsumentenrechte als Mittel der Verhaltenssteuerung von Unternehmen oder die zahlreichen Gerichtsurteile, die inzwischen Tarifverhandlungen und Streikabstimmungen zugleich aufhalten und befeuern. Zahlreiche indirekte Indikatoren belegen die Entwicklung: die steilen Wachstumsraten bei den Rechtsschutzversicherungen, die Vervielfachung der Rechtsanwaltbüros pro 100.000 Einwohner in allen europäischen Ländern, die Ausbreitung amerikanischer „law firms“ (mit etwa achtmal so vielen Beschäftigen wie noch 1990) oder die Zunahme der Verweisungen nationaler Gerichte an den EuGH nach Art. 226 EGV (Kelemen 2011, Kap. 3).

Im Ergebnis zeigt sich also ein enormer Bedeutungszuwachs von nicht-majoritären Institutionen in westlichen Demokratien. Das ist eine demokratierelevante Entwicklung, weil nicht-majoritäre Institutionen andere Legitimationsquellen nutzen als etwa Parlamente. Nicht-majoritäre Institutionen legitimieren sich durch Expertise bzw. durch den Schutz der Individualrechte. Sie stärken mithin die liberalen und schwächen die republikanischen Elemente der Demokratien.

Es ist besonders frappierend, dass diese Verschiebung in der Legitimationsarchitektur politischer Herrschaft zuungunsten originär demokratischer Legitimationsquellen auf große Befürwortung in der Bevölkerung zu stoßen scheint. Es sind erstaunlicherweise diese nicht-majoritären Institutionen, denen ein großes Maß an Vertrauen entgegengebracht wird, während die Institutionen, die der Bevölkerung Mitspracherechte gewähren, äußerst kritisch beäugt werden. Die Frage, mittels welcher institutionellen Verfahren eigentlich das demokratische Prinzip am besten verwirklicht werden kann, erhält anhand der skizzierten empirischen Entwicklung eine normativ äußerst irritierende Antwort: Expertise und Individualrechte gewinnen in den westlichen Demokratien an Bedeutung und dies scheint bei der Bevölkerung auf Gegenliebe zu stoßen. Die traditionelle politikwissenschaftliche Lehrmeinung, wonach Parlamente und Parteien das demokratische Prinzip institutionell am besten verwirklichten, überzeugt jedenfalls nicht mehr ohne Weiteres.

\subsection{Die Erfolge des Autoritarismus}

Ganz entgegen der Mauerfalleuphorie-Prognose von Francis Fukuyama (1989) ist die Geschichte nicht zu Ende gegangen. Dem westlichen Ordnungsmodell der liberalen Demokratie ist inzwischen wieder Konkurrenz erwachsen. Die Aufregung, 
die die chinesischen Wachstumsraten hervorrufen, ist nur vordergründig ökonomischen Ängsten geschuldet. Zum einen wird gerne übersehen, dass derzeit ein Prozent Wachstum des Bruttoinlandsprodukts pro Kopf in Deutschland ungefähr denselben absoluten Zuwachs bedeutet wie zehn Prozent in China. Zum anderen profitieren von einem ökonomisch starken China die deutsche und andere exportorientierte Volkswirtschaften genauso wie von den gleichfalls aufstrebenden großen Volkswirtschaften in mehr oder weniger demokratischen Ländern wie Brasilien und Indien, vor denen wir uns aber offensichtlich viel weniger fürchten.

Viel gewichtiger scheint hingegen die politische Implikation: Spätestens seit der Finanzkrise erwächst der liberalen Demokratie westlicher Provenienz mit China eine ordnungspolitische Konkurrenz, die im Gegensatz zum real existierenden Sozialismus der späten Jahre beides ist: anders und erfolgreich. Sie ist anders, weil sie explizit die Entfaltung ökonomischer Marktdynamiken nicht an die Institutionen der liberalen Demokratie koppelt, sie also die scheinbar unauflösbare Verbindung von Markt und Demokratie infrage stellt. Sie ist erfolgreich, weil die autoritär regierenden Eliten in Ländern wie China und Singapur nicht ohne Weiteres als eigensüchtige Despoten abgetan werden können. Ihre Politik hat eine erkennbare Gemeinwohlkomponente und kann dabei auf erhebliche Erfolge verweisen. $\mathrm{Zu}$ dem sind die Form der Machtausübung und auch die Machtübernahme gleichsam konstitutionell geregelt und weisen eine gewisse Berechenbarkeit auf, ohne dass die individuellen politischen Rechte auch nur annähernd gesichert wären.

Der herausragende Erfolg chinesischer Politik liegt ganz sicher in einer ungekannt schnellen Wohlstandsmehrung begründet. Die Wachstumsraten Chinas sind legendär. Nach IMF-Angaben liegt das durchschnittliche Wachstum des Bruttoinlandsprodukts in den letzten zehn Jahren (2001-2011) bei über zehn Prozent pro Jahr. In nur einer Dekade ist also der Wohlstand Chinas gemessen an diesem Indikator um fast 160 Prozent gestiegen - wie schon in der Dekade davor. Entscheidend ist zudem, dass die Armutsbekämpfung dabei enorme Erfolge erzielt. Gemessen an der nationalen Armutsgrenze sind zwischen 1996 und 2004 ca. 400 Millionen der Armut entkommen. Unter Anwendung des internationalen 2 Dollar-Standards sind es fast 200 Millionen Menschen für den gleichen Zeitraum (für eine Einordnung der unterschiedlichen Berechnungsmethoden vgl. Chen u. Ravallion 2008). Mit anderen Worten: Die Anzahl der Menschen, die in den letzten 15 Jahren in China aus den Fängen absoluter Armut befreit worden sind, ist höher als die entsprechende Zahl in ganz Europa im gesamten 20. Jahrhundert.

Insofern stellt der chinesische Weg, der autoritäre politische Strukturen mit der kontrollierten Einführung von Märkten verbindet, auf stetiges Wachstum und technologischen Fortschritt setzt und effektive Armutsbekämpfung im großen Stil sowie politische Unabhängigkeit verspricht, ein attraktives Entwicklungsmodell dar. Gepaart wird dieses Entwicklungsmodell mit einer alles in allem eher von Vorsicht geprägten Außenpolitik, die zumeist auf Nachahmungsdruck oder gar Missionarismus verzichtet, aber gleichwohl von der Wirkungskraft des eigenen Weges ausgeht (Kissinger 2011). Aus chinesischer Perspektive sind die beschriebenen Erfolge auch nicht Teil einer ,Aufstiegsstory', sondern vielmehr Ausdruck der längst fälligen Rückkehr einer großen Kultur auf die weltpolitische Landkarte. 
Dementsprechend wird in der internationalen Debatte auch bereits das chinesische Modell postuliert - und zwar nicht nur in Afrika. Seit der Schrift eines Goldman Sachs-Mitarbeiters - denen wir schon die Einführung des Konzepts der BRICs in die politische Debatte verdanken - wird über die Ersetzung des Washington Consensus durch den sog. Beijing Consensus gesprochen, der durch die Begriffstrias „Innovation“, „Chaosmanagement“ und „Selbstbestimmung bzw. Nichtintervention" gekennzeichnet sei (Cooper Ramo 2004). ${ }^{15}$ Gleichzeitig konstatieren Größen unseres Faches - wie Joseph Nye - einen beeindruckenden Anstieg der chinesischen „soft power" (vgl. Nye 2010 und auch Cho u. Jeong 2008).

Diesen Entwicklungen entspricht das neue Selbstvertrauen, mit dem die chinesische Führung auftritt. Wurde bis vor Kurzem noch bei jedem Auslandsbesuch betont, man möchte vom Westen lernen, so hat sich inzwischen die Rhetorik verändert: Es wird jetzt die Notwendigkeit gegenseitigen Lernens hervorgehoben. Freilich bleibt das neue chinesische Selbstvertrauen prekär. Die Aufstände in Nordafrika machen deutlich, dass die normative Kraft der individuellen Freiheit und Selbstbestimmung auch und gerade dann fortwirkt, wenn für einen gewissen materiellen Wohlstand gesorgt ist. Die chinesische Regierung registrierte dementsprechend die Entwicklungen in Nordafrika mit höchster Sensibilität und verschärfte die Repressionen gegen die Oppositionellen im eigenen Land. Und damit werden auch die Grenzen des Anstiegs der soft power Chinas offenbar: Innerhalb der westlichen Welt ist sie nach wie vor weitestgehend abwesend.

Dennoch: China, aber auch Singapur und Vietnam zeigen, dass zumindest über einige Jahrzehnte hinweg eine vertretbare Vorstellung des Gemeinwohls befördert werden kann, ohne dass die Machthaber demokratischer Kontrolle unterliegen und ohne dass Individualrechte garantiert werden. Damit wird die insbesondere nach 1989 vertretene Vorstellung von der Alternativlosigkeit der liberalen Demokratie untergraben. Die demokratische Euphorie speiste sich ja 1989 nicht ausschließlich aus der normativen Logik der individuellen Selbstbestimmung, sondern eben auch aus der empirisch begründeten Erwartung, dass Wohlfahrt und Gemeinwohl langfristig am besten nur im Rahmen einer liberalen Demokratie gemehrt werden können. Wenn jetzt China zumindest außerhalb der westlichen Welt als ordnungspolitische Alternative gesehen wird, dann erweist sich diese Annahme als fraglich und damit kehrt die Frage nach der richtigen politischen Ordnung - also die Geschichte in der Terminologie Fukuyamas - wieder auf die weltpolitische Tagesordnung zurück.

Das autoritäre Entwicklungsmodell betont die Autonomie des Kollektivs und dessen Wohlfahrtsgewinne als zentrale Legitimationsquellen. Auch in diesem Fall gewinnen also politische Institutionen an Bedeutung, die sich nicht auf die politische Partizipation und die öffentliche Deliberation als Rechtfertigung der Herrschaftsausübung berufen. Expertise und Wohlfahrtsgewinn, die Steigerung des kollektiven Selbstwertgefühls und „weiche“ Rechenschaftspflichtigkeit sind die entscheidenden Legitimationsressourcen. Diese Legitimationsstrategie geht mit

15 Kritisch dazu Kennedy (2010). Ein erheblicher Teil der Diskussion über den Beijing Consensus bezieht sich auf die Komponente der Selbstbestimmung, die westlichen Vorstellungen von Global Governance widerspricht. Siehe dazu Chan et al. (2008). 
der teilweise expliziten Zurückweisung von Individualrechten und „exzessiver“ politischer Partizipation und Meinungsfreiheit einher.

Dass diese Form der politischen Legitimation recht erfolgreich ist, zeigen die Daten des World Values Survey. Befragt nach dem Vertrauen in die politischen Institutionen des Landes erzielen Länder wie China (mit 80,2 Prozent) und Vietnam (gar mit 91,1 Prozent) hervorragende Werte, die deutlich über dem Durchschnitt der konsolidierten westlichen Demokratien (mit 62 Prozent) liegen (Norris 2011, S. 88). Besonders erstaunlich ist dabei, dass diese andere Form der politischen Legitimation offensichtlich zu einem erheblichen Ausmaß als demokratische Legitimation umgedeutet wird. Gemäß denselben Daten sind in Vietnam 79,4 Prozent und in China 67,1 Prozent der Menschen mit der „demokratischen Performanz ihres Landes zufrieden“, was ungefähr den Werten in den westlichen Demokratien entspricht. Mehr noch: Mit 95 Prozent bzw. 94 Prozent werden in diesen Ländern demokratische Orientierungen grundsätzlich begrüßt (Norris 2011, S. 90-95).

Im Ergebnis wiederholt sich der Befund, der sich auch schon mit Blick auf die westlichen Demokratien abzeichnet: Es lässt sich eine Verschiebung zugunsten von politischen Institutionen beobachten, die de facto auf anderen als demokratischen Legitimationsquellen beruhen und diese Entwicklung findet in den betroffenen Bevölkerungen eine hohes Maß an Zustimmung. Dennoch lässt sich auf Seiten der chinesischen Führung eine gewisse Furcht vor der Ausbreitung der Idee der individuellen und kollektiven Selbstbestimmung beobachten.

\subsection{Die Ausbreitung internationaler politischer Autorität}

Die dritte Entwicklung, die dafür sorgt, dass die Rechtfertigung politischer Herrschaft sich verschiebt, findet jenseits des Nationalstaates statt. Dort hat sich als Reaktion auf die gesellschaftlichen Denationalisierungsprozesse ein dichtes Netz aus internationalen Regelungen und Organisationen entwickelt, die sich sowohl in Qualität als auch in Quantität von den traditionellen internationalen Institutionen abheben (vgl. Zürn 1998). Obgleich viele der neuen transnationalen und internationalen Regelungen längst nicht effektiv im Sinne der Zielerreichung sind, haben sie die politische Institutionenlandschaft doch deutlich verändert.

Das bekannteste Beispiel dafür ist die Europäische Union. Die EU ist längst eine politische Institution geworden, die eigenständig Herrschaft ausübt und Teil der politischen Auseinandersetzung ist. Insofern hat in Europa unbestritten eine politische Einrichtung an Bedeutung gewonnen, die zwar im Kern als legitim und notwendig erachtet wird, sich aber bestenfalls zweitrangig mit Bezug auf die originär demokratischen Legitimationsquellen rechtfertigt. ${ }^{16}$ Die EU stellt mit diesen Merkmalen in der internationalen Institutionenlandschaft keine Ausnahme mehr dar, sondern ist Teil eines allgemeinen Trends.

16 Siehe gleichsam als state of the art und statt vieler: Die Beiträge zur special issue anlässlich des fünfzigsten Jubiläums des Journal of Common Market Studies, die von Walter Mattli and Alec Stone Sweet herausgegeben werden wird und 2012 erscheint. 
Das quantitative Wachstum internationaler Regelungen in einem weit gefassten Verständnis zeigt sich deutlich in der Entwicklung der Anzahl internationaler Abkommen. So stieg die Anzahl aller bei der UN registrierten internationalen Verträge von $8.776 \mathrm{im}$ Jahr 1960 auf $63.419 \mathrm{im}$ Jahr 2010 (United Nations 2010).

Das Entscheidende ist aber, dass die neuen internationalen Regelungen tief in innerstaatliche Angelegenheiten eingreifen und mithin das Konsensprinzip internationaler Politik und damit die Logik staatlicher Souveränität unterlaufen. Zwei Entwicklungen sind für diese qualitative Veränderung verantwortlich (vgl. ausführlicher Zürn 2010). Zum einen umgehen internationale Institutionen das zwischenstaatliche Konsensprinzip, indem Entscheidungen durch eine Form des Mehrheitsstimmrechts bzw. einer informellen Dominanz hegemonialer Mächte getroffen werden. Mehrheitsentscheidungen und die Ausübung der Dominanz durch starke Staaten erhöhen die Handlungsfähigkeit internationaler Institutionen, indem sie das Veto einzelner Staaten aushebeln und Blockaden überwinden. Heute haben grob zwei Drittel aller internationalen Organisationen mit Beteiligung von mindestens einer großen Macht die Möglichkeit, Mehrheitsentscheidungen zu fällen (vgl. Blake u. Payton 2008). Für den Umweltbereich zeigt sich, dass knapp 60 Prozent aller involvierten internationalen Organisationen Mehrheitsentscheidungen für bestimmte Fragen vorsehen, diese aber nur vergleichsweise selten (20,4 Prozent) zur Anwendung kommen (vgl. Breitmeier et al 2006, Kap. 4). Auch wenn das Instrument der Mehrheitsentscheidung in internationalen Verhandlungen weit weniger genutzt wird, als es formal zur Verfügung steht, übt es dennoch auf Vetospieler einen Druck zur Kompromissbereitschaft aus und untergräbt mithin das Konsensprinzip.

Zum anderen delegieren Staaten zunehmend Kompetenzen direkt an internationale Organisationen. So implementieren insbesondere die Weltbank und einige andere internationale Organisationen Politiken selbstständig. Aber auch bei solchen internationalen Normen, die erst durch die Nationalstaaten umgesetzt werden müssen, spielen transnationale und internationale Institutionen eine gewichtige Rolle. Das monitoring der Umsetzung internationaler Normen wird häufig von transnationalen Nichtregierungsorganisationen wie Human Rights Watch übernommen und liegt nicht mehr alleine in der Hand der jeweiligen nationalen Berichterstattung. Falls im Zuge dieser Kontrolle Streitigkeiten über die Normeinhaltung auftreten, ist inzwischen die Delegation der Streitschlichtung an eine dritte Partei zum Normalfall geworden. Die Zunahme entsprechender quasi-gerichtlicher Organe auf der internationalen Ebene führt dabei auch zu de facto gesetzgebenden Momenten - die in jedem Fall in der Rechtssprechung enthalten sind -, ohne dass die Staaten Kontrolle darüber ausüben können (vgl. Bogdandy u. Venzke 2010). Im Jahr 1960 existierten weltweit lediglich 27 gerichtsähnliche Organe; im Jahr 2004 war ihre Anzahl auf 97 gestiegen (vgl. Project on International Courts and Tribunals 2000; Alter 2009). Hinzu kommt die gewachsene Bedeutung internationaler Sekretariate und eigens für die Evaluation internationaler Politiken geschaffener Einrichtungen (Haas u. Stevens 2011; Mitchell 2006). Dabei handelt es sich zumeist um „Wissensagenturen“ wie etwa das International Panel for Climate Change, die die autoritative Deutung des Wissenstandes auf internationaler Ebene vornehmen und damit die Spielräume der nationalen Regie- 
rungen bei der allgemeinen Begründung der eigenen Positionen erheblich einschränken. In der Summe können wir davon ausgehen, dass gut ein Drittel aller internationalen Implementations-, Interpretations- und Bewertungsentscheidungen an eigenständig agierende internationale Organisationen delegiert worden ist (für den Umweltbereich vgl. Green u. Colgan 2010, S. 7).

In dem Maße wie die neuen internationalen Institutionen das Konsensprinzip internationaler Politik untergraben und in innere Angelegenheiten eingreifen, üben sie politische Autorität aus. Damit bedürfen sie aber der politischen Legitimation. Dementsprechend werden sie zum Gegenstand öffentlicher Auseinandersetzung und mit Fragen zur Begründung politischer Herrschaft konfrontiert. So wird der Ausgang internationaler Verhandlungen nicht mehr begrüßt, nur weil sie zu einem Ergebnis geführt haben. Das prozessuale Zustandekommen, der Inhalt der Ergebnisse internationaler Politikprozesse und insbesondere die damit verbundenen Kompetenzzuweisungen bedürfen der Rechtfertigung. Auch für internationale Institutionen wird also inzwischen das "Recht auf Rechtfertigung“ (Forst 2007) eingeklagt. Dafür stehen zahlreiche sogenannte globalisierungskritische Gruppen wie Attac ebenso wie der im Nationalen organisierte Widerstand gegen die Aushöhlung demokratischer Souveränität etwa bei Referenden über die europäische Integration.

Die herrschaftskritische Thematisierung internationaler Institutionen und Verträge vollzieht sich aber nicht allein durch Widerstandsaktivitäten. Opposition erfasst nur einen Teil der gegenwärtigen Auseinandersetzung über internationale Institutionen. Gleichzeitig fordern nämlich viele transnationale Nichtregierungsorganisationen und soziale Bewegungen stärkere internationale und transnationale Organisationen, um den Bedarf an internationaler Regelung zu decken. So treten beispielsweise viele Umweltgruppen für eine zentrale Weltumweltorganisation und eine drastische Verschärfung klimapolitischer Maßnahmen auf der internationalen Ebene ein. Viele Regierungen streben ebenfalls stärkere internationale Regelungen, etwa im Bereich der Finanzmärkte, an. Es zeichnet sich mithin parallel zum wachsenden Widerstand gegen internationale Institutionen die Forderung nach stärkeren internationalen Institutionen ab. Internationale Institutionen werden also politisiert (vgl. Zürn u. Ecker-Ehrhardt 2011).

Die skizzierte Entwicklung lässt sich in ähnlicher Weise auch für transnationale Institutionen beobachten. Im Falle von transnationalen Institutionen werden zum einen bestimmte Kompetenzen, insbesondere der Standardsetzung, an private Einrichtungen delegiert, die global gültige Regeln formulieren sollen und sich mithin gleichfalls der Kontrolle der einzelnen Nationalstaaten entziehen. Beispiele für private Formen transnationaler Governance sind etwa die „International Chamber of Commerce“ oder das „International Accounting Standards Board“ (IASB). In ihrem beeindruckenden Buch „The New Global Rulers“ zeigen Tim Büthe und Walter Mattli in einer Fallstudie über den IASB, wie die Bedeutung solcher Arrangements zugenommen hat:

"The shift of financial rule-making to the IASB is part of a striking and much wider - yet little understood - trend that is the focus of this book: the delegation of regulatory authority from governments to a single international private-sector body that, 
for its area of expertise, is viewed by both public and private actors as the obvious form for global regulation" (2011, S. 5).

Hinzu kommen die zahllosen Verhaltenskodizes („,codes of conduct“) und Zertifizierungssysteme („Forest Stewardship Council“, „Rugmark“ etc.), die sich Unternehmen selbst geben. So hat sich die Zahl von sektoralen und firmenweiten codes of conduct seit Anfang der 1990er-Jahre vervielfacht (Kolk u. Van Tulder 2005).

Eine zweite Form transnationalen Regierens besteht in der gemeinsamen Erbringung von Governance-Leistungen durch wirtschaftliche und/oder gesellschaftliche Akteure in Verbindung mit Staaten innerhalb so genannter public-private partnerships (vgl. z. B. Reinicke u. Deng 2000). Beispiele dafür sind das ICANN, das die Vergabe von Internetadressen regelt, die „Consultative Group on International Agricultural Research“, die „Roll Back Malaria Initiative“ oder das „African Programme for Onchocerciasis Control“ (APOC). In all diesen Fällen erbringen gesellschaftliche Akteure unmittelbar eine Reihe von Governance-Leistungen oder produzieren öffentliche Güter, die von Staaten nicht oder nicht allein bereitgestellt werden können. Dabei sind private Akteure neben der Regelsetzung auch in die Regelüberwachung und -interpretation sowie bei der Um- und Durchsetzung der Governance-Inhalte involviert. Es kann gezeigt werden, dass die Anzahl solcher regulativen Standardsetzungen und die relative Bedeutung der privaten Akteure darin im Laufe der Zeit zugenommen haben (vgl. Abbott u. Snidal 2009, S. 53).

Auch mit Blick auf solche transnationalen Institutionen lassen sich gewisse Politisierungsprozesse beobachten: Wenn diese Art der Autoritätsausübung transparent wird, erfolgt ihre politische Infragestellung. So ist insbesondere in Europa die IASB inzwischen der Adressat politischer Kritik (Nölke 2011) und auch codes of conduct werden ernst genommen und hin und wieder in das Licht der Öffentlichkeit gezerrt (Zürn 2008).

In dem Maße also wie internationale und transnationale Institutionen politische Autorität bzw. Herrschaft ausüben, werden sie zum Gegenstand einer Auseinandersetzung über eine angemessene politische Ordnung. Als autoritätsausübende Institutionen bedürfen sie der Legitimation. Im Allgemeinen beruhen diese internationalen Institutionen auch auf einem bemerkenswerten Maß an Anerkennung. In den konsolidierten Demokratien des Westens wird den Vereinten Nationen mehr politisches Vertrauen entgegengebracht als den nationalen Parteien und Parlamenten (Hay 2007, S. 34). Die verschiedenen Wellen des World Values Survey ${ }^{17}$ zeigen zudem, dass eine Mehrheit der Menschen auf der Welt den Vereinten Nationen vertraut (Furia 2005). Weltweit bewerten 49,2 Prozent die Vereinten Nationen positiv („a great deal“ oder „quite a lot"); diese Zahl sinkt nur geringfügig auf 48,1 Prozent, wenn die Befragten aus den EU-Mitgliedstaaten herausge-

17 Diese Umfragen werden von der World Values Survey Association (2009) regelmäßig durchgeführt und wurden bisher in 97 Nationen erhoben - sie repräsentieren damit 90 Prozent der Weltbevölkerung. Allerdings werden nicht in jeder Erhebungsrunde in allen 97 Ländern Befragungen vorgenommen. 
rechnet werden. Deutlich unter diesen Werten schneiden die Vereinten Nationen eigentlich nur im Mittleren Osten ab (Iran 38,9 Prozent; Irak 13,4 Prozent). In China (66,2 Prozent) und Indien (64 Prozent) liegen die Werte sogar oberhalb derer für Europa. ${ }^{18}$

Das Bild kann durch die Ergebnisse von Datensätzen über die Einschätzung internationaler Institutionen in Deutschland geschärft werden (vgl. Mau 2007; Ecker-Ehrhardt et al. 2008). Demnach schreibt ein erheblicher Anteil der Bevölkerung für die Lösung einer wachsenden Zahl von Problemen internationalen Institutionen eine zentrale Bedeutung zu. 55 Prozent der deutschen Bevölkerung äußern die Ansicht, dass Globalisierungsfolgenprobleme am besten durch internationale Institutionen bewältigt werden können (Mau 2007, S. 190). Eine Analyse von Matthias Ecker-Ehrhardt und Bernhard Weßels (2011) zeigt zudem, dass hinsichtlich dessen, was in der Welt passiert, die Bundesbürgerinnen und Bundesbürger alle betrachteten internationalen Organisationen (EU, Weltbank, IWF, WTO, G8, UN) für deutlich einflussreicher als die Bundesregierung halten. Selbst auf die Entwicklungen in Deutschland weisen zwischen 46 und 53 Prozent der Befragten internationalen Organisationen einen erheblichen Einfluss zu, auch wenn die Bundesregierung - zusammen mit der EU - diesbezüglich an erster Stelle steht. Mit anderen Worten: Die Bevölkerung hält die Lösung von globalisierungsinduzierten Problemlagen durch internationale Institutionen nicht nur für wünschenswert, sie schreibt diesen Institutionen auch realpolitisch bereits einen erheblichen Einfluss zu. Es kann daher kaum noch überraschen, dass die Bevölkerung große internationale Organisationen wie die EU und die Vereinten Nationalen nach ähnlichen Kriterien bemisst wie nationale politische System und ihnen dabei Bewertungen zukommen lässt, die sich erstaunlich wenig von denen nationaler politischer Systeme unterscheiden (Nullmeier et al. 2010, Kap. 5, 7 und 8).

Die in diesen Daten zum Ausdruck kommende Legitimität internationaler Institutionen beruht freilich nicht auf direkter politischer Partizipation der Regelungsbetroffenen oder auf öffentlicher Deliberation. Internationale Institutionen stellen abgesehen von Konsultationsmechanismen für transnationale Nichtregierungsorganisationen (vgl. Tallberg et al. 2011) keine Möglichkeiten für eine direkte politische Partizipation bereit. Internationale Institutionen kennen mit Ausnahme des europäischen Parlaments keine Direktwahlen. Und auch die grenzüberschreitende öffentliche Deliberation ist trotz der skizzierten Politisierungsprozesse internationaler Institutionen kaum entwickelt. Im Kern berufen sich internationale Institutionen zum Zwecke ihrer Legitimation dementsprechend auf ihre Expertise und Sachorientierung sowie teilweise auf den Schutz der Individualrechte und durchweg auf eine hinreichende accountability. Wiederum lässt sich somit beobachten, dass eine Machtverschiebung in der politischen Insti-

18 Die Daten des World Values Surveys decken sich weithegend mit den Daten des PEW Global Attitude Project. Laut dieser Umfragen bewerten weltweit 58 Prozent die Vereinten Nationen als positiv („,very favorable“ oder „,somewhat favorable“). Auch hier sinkt der Wert nur leicht auf 56.5 Prozent, wenn die EU-Mitgliedsstaaten herausgerechnet werden. Deutlich unter diesen Werten liegen Pakistan (17,4 Prozent) und die Palästinensischen Autonomiegebiete (27,1 Prozent). In China $(51,9$ Prozent) und Indien $(47,2)$ liegen die Werte jedoch im Vergleich zum World Values Survey - wenn auch nur geringfügig -- unterhalb der Werte Europas (PEW 2007). 
tutionenlandschaft zu einer Schwächung der originär demokratischen Legitimationsquellen führt. Dennoch scheinen - soweit sich dies aus Umfragedaten ablesen lässt - die auf anderen als demokratischen Legitimationsquellen beruhenden internationalen Institutionen politische Legitimität zu besitzen. Die Beobachtung, dass die Bedeutungsabnahme nicht-majoritärer Legitimationsquellen begrüßt wird, bestätigt sich hier.

Allerdings lassen sich in diesem Fall im Zuge der Politisierung internationaler Institutionen auch Forderungen nach einer Demokratisierung beobachten. $\mathrm{Zu}$ mindest wächst mit dem unterstellten Einfluss auch die Kritik an der Intransparenz, Exklusivität und Selektivität internationaler Institutionen und mithin die Bereitschaft zu aktivem Protest gegen sie. Entlang dieser Kategorien werden internationale Institutionen und die Europäische Union deutlich schlechter eingestuft - im Schnitt um sieben Prozent - als das nationale politische System; trotz der enorm skeptischen Grundeinstellung gegenüber Parteien und Politkern.

\subsection{Das Problem konfligierender Rechtsordnungen}

Selbst wenn es gelänge die politische Autorität internationaler Institutionen trotz aller Schwierigkeiten als eigenständige Ordnungen demokratisch zu legitimieren, bliebe aus demokratietheoretischer Sicht ein weiteres Problem. Das Zusammenspiel unterschiedlich legitimierter politischer Ordnungen bedarf selbst der Regulation. Mit der Pluralisierung von Herrschaftsbereichen entsteht der Bedarf nach Koordination zwischen ihnen. Diese herrschaftsrelevante Koordination politischer Ordnungen kann wiederum nicht ohne Weiteres von demokratischen Prinzipien durchdrungen werden.

Das Problem zeigt sich zunächst im Zusammenspiel von politischen Ordnungen, die auf unterschiedlichen Ebenen angesiedelt sind. Es führt nämlich zur Infragestellung der Letztentscheidungskompetenz. Die liberale Demokratie westlicher Prägung ist in einen Konstitutionalismus mit doppelter Funktion eingebettet: Die Verfassung soll die Macht des Staates und seiner Organe beschränken, sie soll aber auch die Entwicklung einer politischen Vision der entsprechenden Gemeinschaft ermöglichen. Zentrales Merkmal des konstitutionalistischen Denkens ist, dass es einen Ort identifiziert, an dem endgültige Entscheidungen getroffen werden können - die final authority oder Kompetenz-Kompetenz. Der westliche Verfassungsstaat lokalisiert diesen Ort im Nationalen. Optimistische Völkerrechtler sehen hingegen Entwicklungen hin zu einem globalen Konstitutionalismus, bei dem das Völkerrecht Suprematie erlangt. Beide Vorstellungen sind jedoch problembeladen. Der nationale Konstitutionalismus bleibt aufgrund der erheblichen Externalitäten vieler politischer Entscheidungen - also der wachsenden Inkongruenz sozialer und politischer Räume im Zuge der Denationalisierung - ineffektiv und exkludierend. Dem globalen Konstitutionalismus mangelt es hingegen an den sozio-strukturellen Voraussetzungen.

Vor diesem Hintergrund wenden sich Rechtspluralisten wie Nico Krisch (2010) gegen die klassischen konstitutionalistischen Vorstellungen. Sie argumentieren, dass die gegenseitige Anerkennung unterschiedlicher, mit Legitimität ausgestatteter Rechtsordnungen hierarchiefrei erfolgen kann und soll. Sie verweisen dabei 
auch auf emergent erwachsende Rechtspraktiken der gegenseitigen Bezugnahme und Anerkennung unterschiedlicher Gerichte.

Freilich bleibt ein grundlegendes Problem: Was passiert, wenn unterschiedliche Ordnungen zu konfligierenden Auffassungen und Ergebnissen gelangen? Mattias Kumm (2009) arbeitet in seiner Grundlegung des kosmopolitischen Konstitutionalismus Prinzipien heraus, mit denen solche Fälle im Rahmen eines legal reasoning bearbeitet werden können. Das verweist aber erneut auf die Frage nach den angemessenen Quellen der Legitimation. Warum sollen solche Konflikte zwischen internationalen und nationalen Regelungen im rechtlichen Raum entschieden werden? Wäre im Falle eines voll entwickelten Rechtspluralismus nicht gerade die Frage der Koordination unterschiedlicher Rechtsordnungen die Schaltstelle der Herrschaftsausübung und müsste nicht gerade diese demokratisch, d. h. im politischen Raum durch Rekurs auf Partizipation und öffentliche Debatten statt durch rechtliche Prozesse legitimiert werden?

Ähnliche Fragen ergeben sich durch die mangelnde Koordination zwischen unterschiedlichen internationalen politischen Institutionen. Es fehlt auf der internationalen Ebene eine Instanz, die autorisiert ist, die Koordination unterschiedlicher Politiken über Problemfelder hinweg zu übernehmen. Regelungen in einem Problemfeld produzieren immer auch Effekte in anderen Bereichen. Wer erfolgreich mehr Wachstum initiiert, erhöht ceteris paribus auch die $\mathrm{CO}_{2}$-Emissionen. Wer eine bestimmte Gesundheitsanforderung an Produkte stellt, schränkt den Handel mit den Produkten ein, die diese Anforderung nicht erfüllen. Deshalb bedarf es auch der Koordination verschiedener Politiken und Maßnahmen, die auf verschiedenen Ebenen und in verschiedenen Problemfeldern formuliert werden.

In nationalen politischen Systemen wird diese Koordinationsleistung sowohl in Form von formalen Prozeduren aufseiten der Entscheidungsträger, z. B. durch Kabinettsbeschlüsse oder Entscheidungen der Verfassungsgerichte, als auch durch öffentliche Debatten aufseiten der gesellschaftlichen Adressaten mehr schlecht als recht vollzogen. Demgegenüber erweist sich die globale Institutionenstruktur als ein komplexes Patchwork von sich teilweise überlappenden Jurisdiktionen. In diesen Fällen hat jeder Bereich seine eigenen Normen und Regeln entwickelt und die Mitgliedschaften variieren von Bereich zu Bereich. So unterscheidet sich beispielsweise die Mitgliedschaft der Welthandelsorganisation deutlich von der der OECD. Debatten und Diskurse finden fast ausschließlich innerhalb der sektoralen Öffentlichkeiten statt, die die Nebenwirkungen ihres Handelns auf andere gesellschaftliche Problemfelder kaum thematisieren. Zudem gibt es keine institutionalisierten Mechanismen der Koordination unterschiedlicher problemfeldspezifischer Regime, bestenfalls entstehen informelle Mechanismen.

Zwar interagieren die unterschiedlichen internationalen Institutionen und passen sich dabei einander auch kontinuierlich an (Oberthür u. Gehring 2006; Gehring u. Faude 2010). Eine grundwertorientierte Gesamtkoordination bleibt jedoch weitestgehend aus. Da der Koordinationsbedarf aber angesichts quantitativ stark zunehmender internationaler Regulierungen anwächst, scheinen sich funktionale Äquivalente herauszubilden. Insbesondere die G8/20-Treffen erbringen eine gewisse Koordinationsleistung, indem sie dringende Themen identifizieren und allgemeine Zielvorgaben für mehrere internationale Institutionen formu- 
lieren. Auch der UN-Sicherheitsrat übernimmt bei der Abwägung der Ziele Frieden und Menschenrechte eine koordinierende Rolle.

Diese Koordinationsinstitutionen haben allerdings gemeinsam, dass sie nicht zum Zwecke der sektoralen Koordination gegründet worden sind und zudem eine wenig inklusive Mitgliedschaft aufweisen. Ihnen fehlt also ein umfassendes Mandat und es bestehen nur unzureichende Verbindungen zwischen diesen Institutionen und den gesellschaftlichen Regelungsadressaten. Die Koordinationsleistungen erweisen sich daher als beschränkt, zufällig und keinesfalls demokratisch legitimiert.

Im Ergebnis ist festzuhalten, dass sich das Problem konfligierender Rechtsordnungen erst abzeichnet und sich zunächst v. a. durch das Fehlen von Koordinationsleistungen ergibt. Insofern sich freilich erste Konturen der Koordination unterschiedlicher Rechtsordnungen - eine besonders vornehme Form politischer Autorität - abzeichnen, bestätigt sich das hier entwickelte Bild. Es werden neue politische Institutionen gestärkt, die legitimationsbedürftig sind, sich aber nicht originär demokratisch legitimieren (können). Die Frage, ob auch diese Entwicklung von den Betroffenen begrüßt wird, kann aufgrund fehlender Daten nicht beantwortet werden. Es zeigt sich aber auch so, dass die Begründung von Herrschaft und die Rolle des demokratischen Prinzips auch aufgrund dieser vierten demokratierelevanten Entwicklung verstärkt zum Gegenstand der politischen Auseinandersetzung werden sollten.

\section{Das demokratische Paradoxon}

Was heißt dies nun für die Perspektiven des demokratischen Regierens im 21. Jahrhundert? Während wir uns bisher noch auf dem Feld des Deskriptiven bewegt haben, begeben wir uns mit der Beantwortung dieser Frage nun endgültig auf das dünne Eis der Prognose. Die Prognosewarnschilder sind gleich zu Beginn aufgestellt worden. Das weitere Lesen erfolgt also auf eigene Gefahr und ohne Gewähr.

Die beschriebenen Verschiebungen bei der Legitimation politischer Herrschaft legen zwei Implikationen mit Blick auf die Perspektiven demokratischen Regierens im 21. Jahrhundert nahe. Erstens: Die vier Entwicklungen - die Entwertung der majoritären Institutionen der parlamentarischen Demokratie, das Wiederauftreten eines alternativen, nicht-demokratischen Entwicklungsmodells, die Zunahme politischer Herrschaftsausübung auf der internationalen Ebene und die Bedeutungszunahme der Koordination unterschiedlicher Rechtsordnungen - verweisen in der Summe machtvoll auf die Wiederkehr der Frage nach der Legitimation politischer Herrschaft. Die vier Entwicklungen produzieren in der Summe ein Paradox, das sich in öffentlichen Auseinandersetzungen manifestieren wird. Wir sehen einerseits eine fast komplette Universalisierung des demokratischen Prinzips. Demokratie wird weltweit als eine anstrebenswerte politische Ordnung akzeptiert. In allen Ländern, die im World Values Survey berücksichtigt sind, bejahen die Befragten mehrheitlich die Demokratie. Mit Ausnahme von nur zwei Ländern (Kolumbien und Russland) tun dies in jedem Land mehr als 80 Prozent der Befragten. Kaum eine Herrschaftsordnung unterlässt es daher sich zumindest in der Rhetorik als demokratisch legitimiert darzustellen. Andererseits gewinnen aber 
weltweit politische Institutionen an Bedeutung, die sich nicht anhand originär demokratischer Legitimationsquellen rechtfertigen: Zentralbanken, Verfassungsgerichte, „independent agencies“, autoritäre Entwicklungsstaaten wie China, die EU, internationale Institutionen und Gerichtshöfe, die unterschiedliche Rechtsordnungen koordinieren. Solche Institutionen berufen sich je nach Ausprägung auf eine Mischung aus Expertise, dem Schutz der Individualrechte und Rechenschaftspflichtigkeit oder auf die Mehrung von Wohlstand und kollektivem Selbstwertgefühl gepaart mit einer gewissen Rechenschaftspflicht. Die Partizipation der Regelungsbetroffenen und gesellschaftsweite öffentliche Auseinandersetzungen verlieren demgegenüber an Bedeutung.

Erstaunlicherweise findet diese Entwicklung in den Umfragedaten einen enormen gesellschaftlichen Rückhalt. In den stabilen Demokratien gibt es eine gewachsene Unzufriedenheit mit den Kerninstitutionen der nationalen Demokratie, mit Parteien, Parlamenten und auch mit den gewählten Regierungen. Demgegenüber haben die sogenannten nicht-majoritären Institutionen, die dem politischen Prozess entzogen sind, wie etwa die Zentralbanken oder die Verfassungsgerichte, fast überall ein deutlich höheres Ansehen als die demokratischen Kerninstitutionen. Autoritäre Systeme, die wie in China die Armut erfolgreich bekämpfen und die Mindestregeln einer „rule by law“ (im Gegensatz zu „rule of law“) einhalten, scheinen unter Gesichtspunkten der Legitimität fest im Sattel zu sitzen, wiewohl es wenigstens schwache Anzeichen für eine Demokratisierungsbewegung gibt. Und auch die internationalen Institutionen genießen trotz ihrer zunehmenden Politisierung ein erstaunlich hohes Maß an Vertrauen.

Während das Prinzip der Demokratie also nach 1989 fast weltweit Anerkennung gefunden hat, beschleunigt sich seitdem der Verfall des Vertrauens in die demokratischen Kerninstitutionen. Dieses Paradox wird im Ergebnis - so meine erste These - die Frage nach der Legitimation von Herrschaft wieder in den Vordergrund rücken. Wenn die Prognose zutrifft, dass die vier beschriebenen Entwicklungen sich nicht auflösen oder umdrehen, dann lässt sich erwarten, dass die Frage nach der Begründung der Herrschaft unter diesen Bedingungen in das Zentrum politischer Auseinandersetzungen rückt. Oder anders: Die Frage nach der politischen Legitimation wird (wieder) reflexiv. Es geht nicht mehr nur darum, ob sich eine gegebene Herrschaftsordnung vor dem Hintergrund anerkannter Legitimationsmuster rechtfertigen kann, die Legitimationsquellen und deren Mischungsverhältnis werden selbst zum Gegenstand der Auseinandersetzung.

Gleichzeitig stehen enorme Policy-Herausforderungen im Raum: die Energieund Klimafrage etwa oder die Verteilungsfrage in schrumpfenden Gesellschaften, um nur zwei Beispiele zu nennen. Diese und andere Fragen sind drängend und von ihrer Beantwortung hängt ganz erheblich unser Wohlergehen ab. Sie werden aber dennoch die Herrschaftsfrage nicht dauerhaft von der politischen Agenda verdrängen, weil im Zuge ihrer Bearbeitung selbst auch Herrschaftsfragen aufgeworfen und die Grenzen bestehender Formen politischer Herrschaft durch sie offenbar werden. In beiden Fällen privilegiert der institutionelle status quo diejenigen Gruppen und Interessen, die möglicherweise die größten Änderungen vornehmen müssten. Im Falle des Klimawandels sind die größten $\mathrm{CO}_{2}$-Emittenten auch diejenigen, die weltpolitisch als am mächtigsten und einflussreichsten gelten 
können - China und die USA. Im Falle der alternden und schrumpfenden Gesellschaft geraten diejenigen in die Minderheitenpositionen, die das stärkste Interesse an den notwendigen Veränderungen haben - die Erwerbstätigen, die Jungen und zukünftige Generationen.

Bei diesen Themen scheinen die etablierten Demokratien westlicher Prägung zu gegenwartsbezogen und zu wenig zukunftsorientiert zu agieren - neben den genannten Themen gilt das u. a. auch für die Schulden öffentlicher Haushalte und die Bildungsfrage. Diese Fälle verweisen auf ein neues Verhältnis von Mehrheitsentscheidung und Problemlösung. In der sozialen Frage, die das 20. Jahrhundert geprägt hat, konnte über die Etablierung von Mehrheitsentscheidungen eine Problemlösung erreicht werden. Die kurzfristigen Interessen der Mehrheit deckten sich mit einem langfristig gedachten gesellschaftlichen Gemeinwohl. Genau dieses Verhältnis scheint sich in Teilen verkehrt zu haben: Die Interessen der Mehrheit sind partikulare Bestandsinteressen auf Kosten des Gemeinwohls und zukünftiger Generationen.

Damit greift die Vorstellung, dass die effizienzsteigernde Regulation nicht-majoritären Institutionen überlassen werden kann, solange die zentralen Verteilungsfragen in der Hand majoritärer Institutionen bleibt (vgl. etwa Majone 1996), nicht mehr. Gerade bei den großen, zukunftsrelevanten Fragen der Umverteilung nimmt der Ruf nach nicht-majoritären politischen Institutionen zu, weil den etablierten Mehrheitsverfahren die Kapazität zur Problemlösung zunehmend abgesprochen wird. Kurz und gut: Auch die zentralen Policyprobleme der Gegenwart und der Zukunft verweisen nachdrücklich auf die Herrschaftsfrage. Im Ergebnis könnte dies den Prozess verstärken, wonach die demokratische Legitimation gegenüber anderen Begründungsprinzipien gleichsam in die Defensive gerät.

Man mag nun einwenden, dass jede Form der politischen Herrschaft - also die Einschränkung von individuellen Freiheitsrechten - letztlich nur durch das Prinzip der Selbstbestimmung gerechtfertigt werden kann und mithin alle legitimen politischen Entscheidungsverfahren demokratisch zu verankern sind. Demnach macht erst die Verbindung von majoritären Entscheidungsverfahren in der Legislative und Exekutive mit nicht-majoritären Entscheidungsmodi (wie in der Judikative) eine demokratische Rechtfertigung möglich. Normativ mag eine solche monistische Legitimationskonzeption überzeugend sein. Empirisch betrachtet scheint das normative Argument aber nicht alle zu überzeugen (vgl. auch Sørensen 2011). Während einerseits der Schutz der Individualrechte und einfache Formen der Rechenschaftspflichtigkeit v. a. in den westlichen Demokratien und in internationalen Institutionen auf Kosten der majoritären Verfahren systematisch an Gewicht zu gewinnen scheinen, wird auf der anderen Seite auf die nationale Autonomie und das Selbstwertgefühl der Gemeinschaft sowie auf erfolgreiche, technokratisch begründbare Politikergebnisse - ohne jegliche demokratische Verankerung - als Legitimationsquelle verwiesen. Im Ergebnis könnte also eine Situation entstehen, in der sich ein zunehmend demokratiearmer Liberalismus westlicher Prägung und ein moderner, aber undemokratischer Autoritarismus, der sich v. a. in Teilen Asiens Bahn bricht, als zentrale Legitimationsmuster von politischer Herrschaft gegenüberstehen. Das demokratische Prinzip könnte dazwischen zer- 
rieben werden. Insofern korrespondiert die Beförderung des Gemeinwohls auf Kosten der Individualrechte in China, Singapur und Vietnam ungut mit der individualrechtlichen Liberalisierung westlicher Demokratien.

Vor diesem Hintergrund kann es auch nicht überraschen, dass die Versuche zunehmen, diese empirischen Entwicklungen in einer rekonstruktiven Logik normativ zu begründen. Dies wird besonders in den Arbeiten von Pierre Rosanvallon deutlich (2011a; 2011b). Dort wird ein neues Zeitalter der Legitimität gefordert, in dem Konzepte wie Unparteilichkeit, Reflexivität und Nähe legitimierende Kraft gewinnen. Sie könnten das Prinzip des allgemeinen Willens in vielen Situationen besser verkörpern als die Mehrheitsentscheidung. Soweit möchte ich nicht gehen. Denn es lassen sich in der Tat gute normative Argumente dafür ausmachen, dass die legitimatorische Kraft der Expertise und der Individualrechte, aber auch die der kollektiven Wohlfahrtserfolge von einer Einbettung in originär demokratische Verfahren abhängig sind (Habermas 1994; Möllers 2008, Kap. 2). Ein ähnliches Argument lässt sich auch empirisch machen. Ein Verfassungsgericht, das nicht hinreichend in mehrheitsdemokratische Verfahren eingebettet ist, könnte sich auf Dauer als wenig vertrauenserweckend erweisen. Zumindest scheint mir aber die Neubewertung und Umgewichtung unterschiedlicher Legitimationsstrategien nur dann greifen zu können, wenn die reflexive Auseinandersetzung darüber in einer einigermaßen herrschaftsfreien öffentlichen Debatte erfolgt. Jedenfalls könnte die fortschreitende Entkoppelung nicht-majoritärer Legitimationsquellen von den originär demokratischen dazu führen, dass den alternativen Legitimationsquellen langfristig der Boden entzogen wird.

Dafür spricht insbesondere, dass dort, wo diese Entkoppelung komplett vorliegt - wie etwa in China - oder aufgrund sehr langer Legitimationsketten besonders weit fortgeschritten ist - wie im Falle der internationalen Institutionen - Gegenbewegungen zu beobachten sind. Insofern ist die Politisierung internationaler Institutionen möglicherweise Ausdruck einer breiteren Entwicklung: der Politisierung der Auseinandersetzung zwischen unterschiedlichen Legitimationsquellen. Die identifizierten Gewichtsverschiebungen in der Nutzung unterschiedlicher Legitimationsquellen verweisen also nicht zwingend auf einen unaufhaltsamen Siegeszug der Expertise und der Individualrechte über Partizipation und öffentlichen Diskurs als Legitimationsquellen politischer Herrschaft. Sie verweisen aber darauf, dass die Frage nach der angemessenen Legitimation politischer Herrschaft Teil der politischen Auseinandersetzung wird.

Gleichwohl: Die originär demokratischen Prinzipien werden in ihrer hegemonialen Stellung herausgefordert. Es sind nicht zuletzt diese Auseinandersetzungen über die Begründung politischer Herrschaft, die - so die Prognose - die kommenden Jahrzehnte mitprägen werden. Die Antwort auf die Frage nach den Perspektiven des demokratischen Regierens im 21. Jahrhundert ist also eine ambivalente: Während die Frage nach der angemessenen Herrschaftsbegründung - also ein in seiner Reflexivität per se demokratisches Anliegen - an Bedeutung gewinnt, verliert möglicherweise die rein liberal-demokratische Antwort auf diese Frage an Überzeugungskraft. 


\section{Statt einer Zusammenfassung: Perspektiven der Politikwissenschaft}

Was heißt das schließlich für die Politikwissenschaft? Es heißt zunächst, dass die Hinwendung der Politikwissenschaft zu Policy-Fragen, die in den 1980er-Jahren als Professionalisierungsprozess gesehen wurde, wie Wilhelm Bleek in seiner Geschichte der Politikwissenschaft in Deutschland zeigt, Ausdruck einer Ausdifferenzierung war, nicht aber eine thematische Umorientierung darstellte (2001, Kap. 10). Die Polity- und Politics-Dimensionen der Politikwissenschaft sind längst zurückgekehrt, ohne dass das Fach deswegen entprofessionalisiert worden wäre.

Damit verbindet sich aber keinesfalls die Rückkehr zur Demokratiewissenschaft der Gründungsväter der Disziplin. Es ist gerade die realweltliche Infragestellung der Prinzipien westlicher Demokratien, die unsere Forschung treiben wird und nicht die Gewissheit der Überlegenheit der parlamentarischen Demokratie, die der Gründung unserer Vereinigung im Februar 1951 zugrunde lag. ${ }^{19}$ In diesem Sinne steht uns also nicht die Rückkehr zur Demokratiewissenschaft, sondern möglicherweise der Aufbruch zur Legitimitätswissenschaft bevor.

Die Professionalisierung und der relative Bedeutungsgewinn der Politikwissenschaft in den letzten Jahrzehnten sind anhand der üblichen Indikatorik - Studierende, Stellen, Drittmittel und internationale Rezeption - mehrfach beschrieben worden. Selbst in der Öffentlichkeit steht die Politikwissenschaft inzwischen ganz gut da. Die Äußerungen Helmut Schmidts illustrieren diesen Wandel nachdrücklich. 1968 hatte er sich noch verheerend über das Fach geäußert. Ich zitiere: „Wir haben zu viel Soziologen und Politologen. Wir brauchen viel mehr Studenten, die sich für anständige Berufe entscheiden, die der Gesellschaft auch nützen “ (zitiert nach Bleek 2001, S. 365). In seiner Rede zum 100sten Geburtstag der KaiserWilhelm-Gesellschaft hört sich das schon anders an. Mehrfach skizziert er dabei originär politikwissenschaftliche Themen als die großen bevorstehenden wissenschaftlichen Herausforderungen. Und die einzige Disziplin, die in seiner Laudatio auf die Wissenschaft ihr Fett abbekommt, ist heute die Ökonomie, die sich in den letzten Jahrzehnten mit allem, aber nicht mit Ruhm bekleckert habe (Schmidt 2011). Nur eine langfristige Prognose eines Ökonomen habe Bestand - die von John Maynard Keynes: "In the long run, we are all dead."

Im Konzert der Wissenschaften könnte sich mithin der beschriebene Bedeutungszuwachs der Politikwissenschaft fortsetzen oder zumindest stabilisieren. Sicherlich steht die Dominanz der Lebenswissenschaften als allgemeine Leitwissenschaft nicht zur Debatte. Innerhalb der Sozialwissenschaften scheinen mir aber die beschriebenen Entwicklungen das Profil der Politikwissenschaft zu akzentuieren. Nur die Politikwissenschaft scheint das konzeptionelle Handwerkszeug zu haben, um Legitimationsprozesse empirisch sowohl auf der Einstellungsebene, auf der Ebene von diskursiven Legitimationsprozessen als auch auf der institutionellen Ebene analysieren und sie zugleich normativ an Grundfragen der politischen Theorie rückbinden zu können. Sie hat das Potenzial ,Füchsewissenschaft ${ }^{`}$ zu sein,

19 Für Hans Kastendiek war die deutsche Politologie sogar eine „Wissenschaft, die die spezifische Form der Demokratie propoagierte, welche sich in Westdeutschland konstituiert hatte " 1975 , S. 53). 
die - um auf Tetlocks Analyse zurück zu kommen - weder den deduktiven oder subsumtiven Logiken großer Teile der Sozialwissenschaften folgt, noch datengetrieben die relevanten Fragen vergisst und trotzdem theorie- und methodenbewusst arbeitet. Hinzu kommt, dass der Politikwissenschaft der methodologische Nationalismus weniger eingeschrieben zu sein scheint als anderen sozialwissenschaftlichen Disziplinen und sie damit zentrale Fragen zukünftiger Legitimationsprozesse - nämlich das Zusammenspiel der nationalen und der internationalen Ebene - besser erfassen kann. Mir scheint also die Fähigkeit der Politikwissenschaft, thematisch und theoretisch vielseitig anschlussfähig zu sein, nicht primär eine Gefahr für die Identität des Faches, sondern vielmehr eine große Chance für die Bedeutung des Faches im Konzert der sozialwissenschaftlichen Disziplinen zu sein.

Dennoch möchte ich mit einem warnenden Plädoyer enden. Die Politikwissenschaft täte gut daran, Forschung zu den skizzierten Fragen auf eine verbesserte und neue Datenbasis zu stellen. Während etwa die Mikrosoziologie entscheidende Fortschritte durch die vermehrte Entwicklung und Verwendung von Panel-Daten in DFG-Langzeitprogrammen macht und die Verbindung von Ökonomie und Psychologie in der behavioral economics durch Laborexperimente enorm voranschreitet, ist die Datenbasis zu den von mir skizzierten Entwicklungen vergleichsweise mager. Am besten schaut es hierbei noch mit Blick auf die Entwicklung westlicher Demokratien aus. Schon wenn es um die Entwicklung nationaler politischer Systeme außerhalb der OECD-Welt geht, verschlechtert sich die Datenlage drastisch. Und systematisch entwickelte Datensätze zur Entwicklung politischer Autorität jenseits des Nationalstaates sowie den mit ihr verbundenen Legitimationsanforderungen und -strategien fehlen völlig.

Der thematische und konzeptionelle Vorteil der Politikwissenschaft wird mittelfristig ungenutzt bleiben, wenn es nicht gelingt, unsere Wissenschaft auf eine verbesserte Datenbasis zu stellen. Nur wenn die politikwissenschaftliche ,Füchseforschung' sich als methodisch stark und überzeugend erweist, wird sie der deduktiv-modellhaften, nicht selten auch empiriefernen und tendenziell imperialen ,Igelforschung' widerstehen können. Oder anders gesagt: Nur weil Tetlocks Arbeit selbst auf einer so überzeugenden Datenbasis beruht und methodisch hochwertig vorgeht, kann sie die Grenzen der ,Igelei‘ aufzeigen. Das Buch zeigt geradezu exemplarisch, dass datengestützte und reflexive Wissenschaft kein Widerspruch zu sein braucht. Insofern kann und sollte es der Politikwissenschaft als Vorbild dienen.

\section{Literatur}

Abbott, Kenneth W., und Duncan Snidal. 2009. The Governance Triangle: Regulatory Standards Insitutions and the Shadow of the State. In The Politics of Global Regulation, Hrsg. Walter Mattli und Ngaire Woods, 44-88. Princeton: Princeton University Press.

Alter, Karen J. 2009. The European Court's Political Power. Selected Essays. Oxford: Oxford University Press.

Anderson, Benedict. 1983. Imagined Communities: Reflections on the Origin and Spread of Nationalism. London: Verso. 
Beetham, David. 1991. The Legitimation of Power. Atlantic Highlands: Humanities Press International.

Berlin, Isaiah. 1953. The Hedgehog and the Fox: An Essay on Tolstoy's View of History. London: Weidenfeld \& Nicolson.

Blake, Daniel, und Autumn Payton. 2008. Voting Rules in International Organizations: Reflections of Power or Facilitators of Cooperation? Beitrag präsentiert auf der 49th ISA Annual Convention, 26.-29.3.2008, San Francisco.

Bleek, Wilhelm. 2001. Geschichte der Politikwissenschaft in Deutschland. München: C. H. Beck.

Bogdandy, Armin v., und Ingo Venzke. 2010. Zur Herrschaft internationaler Gerichte: Eine Untersuchung internationaler öffentlicher Gewalt und ihrer demokratischen Rechtfertigung. Zeitschrift für ausländisches öffentliches Recht und Völkerrecht 70:1-49.

Breitmeier, Helmut, Oran R. Young und Michael Zürn. 2006. Analyzing Environmental Regimes. From Case Study to Database. Cambridge: MIT Press.

Buchstein, Hubertus. 2009. Demokratie und Lotterie: Das Los als politisches Entscheidungsinstrument von der Antike bis zur EU. Frankfurt a. M.: Campus Verlag.

Bühlmann, Marc, Wolfgang Merkel und Lisa Müller. 2011. New Democracy Barometer shows how democratic the thirty best democracies are. NCCR Democracy Newsletter February 2011:1-4.

Büthe, Time, und Walter Mattli. 2011. The New Global Rulers: The Privatization of Regulation in the World Economy. Princeton: Princeton University Press.

Chan, Lai-Ha, Pak K. Lee und Gerald Chan. 2008. Rethinking Global Governance: A China Model in the Making? Contemporary Politics 14:3-19.

Chen, Shaohua, und Martin Ravallion. 2008. China is Poorer than we Thought, But No Less Successful in the Fight Against Poverty. Policy Research Working Paper 4621. Washington: World Bank Development Research Group.

Cho, Young N., und Jong H. Jeong. 2008. China's Soft Power: Discussions, Resources, and Prospects. Asian Survey 48:453-472.

Cooper Ramo, Joshua. 2004. The Beijing Consensus. London: Foreign Policy Centre.

Crouch, Colin. 2008. Postdemokratie. Frankfurt a. M.: Suhrkamp.

Cukierman, Alex. 2008. Central Bank Independence and Monetary Policymaking Institutions - Past, Present and Future. European Journal of Political Economy 24:722-736.

Dahl, Robert A. 1989. Democracy and its Critics. New Haven: Yale University Press.

Easton, David. 1975. A Re-Assessment of the Concept of Political Support. British Journal of Political Science 5:435-457.

Ecker-Ehrhardt, Matthias, Wolfgang Merkel, Bernhard Weßels und Michael Zürn. 2008. Denationalisierung von Problemwahrnehmungen. Repräsentative Bevölkerungsumfrage. Berlin: Wissenschaftszentrum Berlin für Sozialforschung.

Ecker-Ehrhardt, Matthias, und Bernhard Weßels. 2011. Input- oder Output-Politisierung internationaler Organisationen? Der kritische Blick der Bürger auf Demokratie und Leistung. In Gesellschaftliche Politisierung und internationale Institutionen, Hrsg. Michael Zürn und Matthias Ecker-Ehrhardt. Berlin: Suhrkamp (im Erscheinen).

Forst, Rainer. 2007. Das Recht auf Rechtfertigung. Elemente einer konstruktivistischen Theorie der Gerechtigkeit. Frankfurt a. M.: Suhrkamp.

Fukuyama, Francis. 1989. The End of History. The National Interest 16:3-18. 
Furia, Peter A. 2005. Global Citizenship, Anyone? Cosmopolitanism, Privilege and Public Opinion. Global Society 19:331-359.

Gehring, Thomas, und Benjamin Faude. 2010. Institutional Ecology and the Evolution of Interlocking Structures of International Governance. The Trade and Environment Overlap. Beitrag präsentiert auf der 51st Annual Convention der International Studies Association, 17.2.2010, New Orleans.

Gilardi, Fabrizio. 2008. Delegation in the Regulatory State: Independent Regulatory Agencies in Western Europe. Cheltenham: Edward Elgar.

Grant, Ruth W., und Robert O. Keohane. 2005. Accountability and Abuses of Power in World Politics. American Political Science Review 99:29-43.

Green, Jessica F., und Jeff Colgan. 2010. Is There Really a Power Shift? Delegating Authority in Global Environmental Politics. Beitrag präsentiert auf der 7th Pan-European Conference on IR der Standing Group on International Relations, 9.-11.9.2010, Stockholm.

Haas, Peter M., und Casey Stevens. 2011. Organized Science, Usable Knowledge and Multilateral Environmental Governance. In Governing the Air: The Dynamics of Science, Policy, and Citizen Interaction, Hrsg. Rolf Lidskog und Göran Sundqvist. Cambridge: MIT Press (im Erscheinen).

Habermas, Jürgen. 1973. Legitimationsprobleme im Spätkapitalismus. Frankfurt a. M.: Suhrkamp.

Habermas, Jürgen. 1994. Faktizität und Geltung. Beiträge zur Diskurstheorie des Rechts und des demokratischen Rechtsstaats. Frankfurt a. M.: Suhrkamp.

Halberstam, Daniel. 2009. Local, Global, And Plural Constitutionalism: Europe Meets The World. Public Law and Legal Theory Working Paper No. 176. Ann Arbor: University of Michigan Law School.

Hay, Colin. 2007. Why We Hate Politics. Cambridge: Polity Press.

Hirschl, Ran. 2004. Towards Juristocracy: The Origins and Consequences of the New Constitutionalism. Harvard: Harvard University Press.

Hurd, Ian. 2007. After Anarchy. Legitimacy and Power in the United Nations Security Council. Princeton: Princeton University Press.

Infratest dimap. 2009. Vertrauen der Bürger in die Politik gestiegen: Infratest dimap Erhebung zum Institutionenvertrauen. http://www.infratest-dimap.de/uploads/media/2009_03_18_Infratest-dimap_Institutionenvertrauen.pdf. Zugegriffen: 8. September 2011.

Jordana, Jacint, David Levi-Faur und Xavier Fernández i Marín. 2011. The Global Diffusion of Regulatory Agencies: Channels of Transfer and Stages of Diffusion. Comparative Political Studies: online first. doi: 10.1177/0010414011407466.

Kastendiek, Hans. 1975. Desintegration einer Integrationswissenschaft. Konstituierung und Wandel der westdeutschen Politologie. In Kritik der Politischen Wissenschaften I, Hrsg. Bernhard Blanke, Ulrich Jürgens und Hans Kastendiek, 27-125. Frankfurt a. M.: Campus Verlag.

Kelemen, R. Daniel. 2011. Eurolegalism: The Transformation of Law and Regulation in the European Union. Cambridge: Harvard University Press.

Kennedy, Scott. 2010. The Myth of the Beijing Consensus. Journal of Contemporary China 19:461-477. 
Kissinger, Henry A. 2011. China. Zwischen Tradition und Herausforderung. München: C. Bertelsmann Verlag.

Kneip, Sascha. 2009. Verfassungsgerichte als demokratische Akteure: Der Beitrag des Bundesverfassungsgerichts zur Qualität der bundesdeutschen Demokratie. Baden-Baden: Nomos.

Kolk, Ans, und Rob Van Tulder. 2005. Setting New Global Rules? TNCs and Codes of Conduct. Transnational Corporations 14:1-27.

Krisch, Nico. 2010. Beyond Constitutionalism: The Pluralist Structure of Postnational Law. Oxford: Oxford University Press.

Kumm, Matthias. 2009. The Cosmopolitan Turn in Constitutionalism: On the Relationship Between Constitutionalism In and Beyond the State. In Ruling the World: International Law, Global Governance, Constitutionalism, Hrsg. Jeffrey L. Dunoff und Joel P. Trachtman, 258-325. Cambridge: Cambridge University Press.

Lipset, Seymour M. 1960. Political Man: The Social Bases of Politics. London: Heinemann. Majone, Giandomenico. 1996. Regulating Europe. London: Routledge.

Mau, Steffen. 2007. Transnationale Vergesellschaftung. Die Entgrenzung sozialer Lebenswelten. Frankfurt a. M.: Campus Verlag.

Mitchell, Ronald B. (Hrsg.). 2006. Global Environmental Assessments: Information and Influence. Cambridge: MIT Press.

Möllers, Christoph. 2008. Die drei Gewalten. Legitimation der Gewaltengliederung in Verfassungsstaat, Europäischer Integration und Internationalisierung. Weilerswist: Velbrück Verlag.

Mouffe, Chantal. 2007. Über das Politische wider die kosmopolitische Illusion. Frankfurt a. M.: Suhrkamp.

Nölke, Andreas. 2011. Die gesellschaftliche Politisierung transnationaler privater Governance: Der Fall des International Accounting Standards Board. In Gesellschaftliche Politisierung und internationale Institutionen, Hrsg. Michael Zürn und Matthias EckerEhrhardt. Berlin: Suhrkamp (im Erscheinen).

Norris, Pippa. 2011. Democratic Deficit: Critical Citizens Revisited. Cambridge: Cambridge University Press.

Norwegian Social Science Data Services. 2011. European Social Survey Education Net. http://essedunet.nsd.uib.no/. Zugegriffen: 30. September 2011.

Nullmeier, Frank, Dominika Bigeon, Martin Nonhoff, Henning Schmidtke und Steffen Schneider (Hrsg.). 2010. Prekäre Legitimitäten: Rechtfertigung von Herrschaft in der postnationalen Konstellation. Frankfurt a. M.: Campus Verlag.

Nye, Joseph S., Jr. 2010. American and Chinese Power after the Financial Crisis. The Washington Quarterly 33:143-153.

Oberthür, Sebastian, und Thomas Gehring (Hrsg.). 2006. Institutional Interaction in Global Environmental Governance: Synergy and Conflict among International and EU Policies. Cambridge: MIT Press.

Offe, Claus. 1972. Strukturprobleme des kapitalistischen Staates. Aufsätze zur politischen Soziologie. Frankfurt a. M.: Suhrkamp.

PEW. 2007. 47-Nation Survey Conducted April 2 - May 28, 2007. http://pewglobal.org/ category/data-sets/. Zugegriffen: 21. September 2010.

PIREDEU. 2010. European Election Study 2009. http://www.piredeu.eu/public/EES2009. asp. Zugegriffen: 15. September 2011. 
Project on International Courts and Tribunals. 2000. PICT Research Matrix. http://www. pict-pcti.org/matrix/matrixintro.html. Zugegriffen: 16. April 2010.

Putnam, Robert D. 2000. Bowling Alone: The Collapse and Revival of American Community. New York: Simon \& Schuster.

Rapaport, Orit, David Levi-Faur und Dan Miodownik. 2009. The Puzzle of the Diffusion of Central-Bank Independence Reforms: Insights from an Agent-Based Simulation. The Policy Studies Journal 37:695-716.

Reinicke, Wolfgang H., und Francis M. Deng. 2000. Critical Choices. The United Nations, Networks, and the Future of Global Governance. Ottawa: International Development Research Centre.

Rosanvallon, Pierre. 2011a. Democratic Legitimacy: Impartiality, Reflexivity, Proximity. Princeton: Princeton University Press.

Rosanvallon, Pierre. 2011b. The Metamorphoses of Democratic Legitimacy: Impartiality, Reflexivity, Proximity. Constellations 18:114-123.

Scharpf, Fritz W. 1991. Die Handlungsfähigkeit des Staates am Ende des zwanzigsten Jahrhunderts. Politische Vierteljahresschrift 32:621-634.

Scharpf, Fritz W. 1999. Regieren in Europa: Effektiv und demokratisch? Frankfurt a.M.: Campus.

Scharpf, Fritz W., Bernd Reissert und Fritz Schnabel. 1976. Politikverflechtung: Theorie und Empirie des kooperativen Föderalismus in der Bundesrepublik. Regensburg: Scriptor.

Schmidt, Helmut. 2011. Verantwortung der Forschung im 21. Jahrhundert. http://www. mpg.de/print/990353. Zugegriffen: 8. September 2011.

Shapiro, Martin. 1997. The Problem of Independent Agencies in the United States and the European Union. Journal of European Public Policy 4:276-291.

Shapiro, Martin. 2005. "Deliberative," "Independent” Technocracy v. Democratic Politics: Will the Globe Echo the E. U.? Law and Contemporary Problems 68:341-356.

Sørensen, Georg. 2011. A Liberal World Order in Crisis: Choosing between Imposition and Restraint. Ithaca: Cornell University Press.

Stone Sweet, Alec. 2000. Governing with Judges: Constitutional Politics in Europe. Oxford: Oxford University Press.

Tallberg, Jonas, Thomas Sommerer und Theresa Squatrito. 2011. Transnational Access to International Organizations: Change and Continuity, 1950 - 2010. Beitrag präsentiert beim Workshop "Institutional Dynamics in World Politics: Explaining Variation in the Scope, Pace, and Direction of International Institutional Change”, 7.4.2011, Berlin: Wissenschaftszentrum Berlin für Sozialforschung.

Tamanaha, Brian Z. 2004. On the Rule of Law: History, Politics, Theory. Cambridge: Cambridge University Press.

Tetlock, Philip E. 2005. Expert Political Judgment: How Good Is It? How Can We Know? Princeton: Princeton University Press.

United Nations. 2010. United Nations Treaty Collection. http://treaties.un.org/Home.aspx. Zugegriffen: 25. May 2010.

Weber, Max. 1968. Wirtschaft und Gesellschaft. Grundriß der Verstehenden Soziologie. Tübingen: Mohr Siebeck. 
World Values Survey Association. 2009. World Values Survey 1981-2008 Official Aggregate v.20090901. http://www.wvsevsdb.com/wvs/WVSData.jsp?Idioma=I. Zugegriffen: 15. September 2011.

Zürn, Michael. 1998. Regieren jenseits des Nationalstaats. Globalisierung und Denationalisierung als Chance. Frankfurt a. M.: Suhrkamp.

Zürn, Michael. 2008. The Politicization of Economization? On the Current Relationship and NGOs. In Handbook of Research on Global Governance Citizenship, Hrsg. Andreas G. Scherer und Guido Palazzo, 293-311. Cheltenham: Edward Elgar.

Zürn, Michael. 2010. Global Governance as Multi-level Governance. In Handbook on Multi-Level Governance, Hrsg. Henrik Enderlein, Sonja Wälti und Michael Zürn, 8099. Cheltenham: Edward Elgar.

Zürn, Michael, und Matthias Ecker-Ehrhardt (Hrsg.). 2011. Gesellschaftliche Politisierung und Internationale Institutionen. Berlin: Suhrkamp (im Erscheinen).

\section{Autorenangaben:}

Prof. Dr. Michael Zürn,

Direktor der Abteilung Transnationale Konflikte und Internationale Institutionen am Wissenschaftszentrum Berlin für Sozialforschung (WZB) und Professor für Internationale Beziehungen an der Freien Universität Berlin, Wissenschaftszentrum Berlin für Sozialforschung, Reichpietschufer 50, 10785 Berlin, zuern@wzb.eu 